\title{
A nonlinear, 3D fluid-structure interaction problem driven by the time-dependent dynamic pressure data: a constructive existence proof
}

\author{
BORIS MUHA* AND SUNČICA ČANIĆ* \\ Dedicated to Marshall Slemrod for his 70th birthday
}

\begin{abstract}
We study a 3D fluid-structure interaction (FSI) problem between an incompressible, viscous fluid modeled by the Navier-Stokes equations, and the motion of an elastic structure, modeled by the linearly elastic cylindrical Koiter shell equations, allowing structure displacements that are not necessarily radially symmetric. The problem is set on a cylindrical domain in $3 \mathrm{D}$, and is driven by the time-dependent inlet and outlet dynamic pressure data. The coupling between the fluid and the structure is fully nonlinear (2-way coupling), giving rise to a nonlinear, moving-boundary problem in 3D. We prove the existence of a weak solution to this 3D FSI problem by using an operator splitting approach in combination with the Arbitrary Lagrangian Eulerian mapping, which satisfies a geometric conservation law property. We effectively prove that the resulting computational scheme converges to a weak solution of the full, nonlinear 3D FSI problem.
\end{abstract}

AMS 2000 SUBJECT ClASSIFICATIONS: 35R37, 35M33.

KEYWORDS AND PHRASES: Fluid-structure interaction, nonlinear movingboundary problem, existence of a solution.

\section{Introduction}

This manuscript was motivated by a conversation with Marshall Slemrod. Marshall suggested to study a fluid-structure interaction problem between an incompressible, viscous fluid and an elastic structure, motivated by applications in blood flow and cardiovascular disease, in which the cylindrical

${ }^{*}$ The research of Muha was supported in part by NSF under grant DMS-1311709. The research of Čanić was supported in part by NSF under grants NIGMS DMS1263572, DMS-1318763, DMS-1311709, DMS-1262385, and DMS-1109189. 


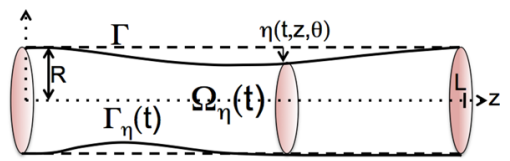

Figure 1: Domain sketch and notation.

fluid domain is not necessarily radially symmetric, since cardiovascular diseases do not typically respect the mathematical property of axial symmetry. The present manuscript is a step in this direction. We study a full 3D fluid-structure interaction (FSI) problem between an incompressible, viscous fluid modeled by the Navier-Stokes equations, and the motion of an elastic structure, modeled by the linearly elastic cylindrical Koiter shell equations, allowing structure displacements that are not necessarily radially symmetric. The problem is set on a cylindrical domain in $3 \mathrm{D}$, and is driven by the time-dependent inlet and outlet dynamic pressure data. See Figure 1. The coupling between the fluid and the structure is fully nonlinear (2-way coupling), giving rise to a nonlinear, moving-boundary problem in 3D. This model problem is a good approximation of blood flow in elastic human arteries. We prove the existence of a weak solution to this 3D FSI problem by using nontrivial extensions of the ideas based on an operator splitting approach that has been implemented in computational solvers applied to $2 \mathrm{D}$ radially symmetric cases (see e.g., [30, 7, 43, 6, 31, 40]).

This is an extension of our earlier results in which the existence of a weak solution to a $2 \mathrm{D}$ problem respecting radial symmetry was proved [43]. The novelties of the present work include dealing with a Koiter shell model in which the shell displacement depends on both the axial, as well as the azimuthal variable, dealing with the lower regularity of the fluid-structure interface which is not necessarily Lipschitz, making sense of the trace of the fluid velocity at the fluid-structure interface, and using appropriate compactness arguments in 3D that provide the existence of a weak solution. Another difference with the $2 \mathrm{D}$ radially symmetric case is in the construction of the appropriate Arbitrary Lagrangian Eulerian (ALE) mapping to deal with the motion of the fluid domain. An ALE mapping had to be constructed to satisfy the so called geometric conservation law property, see [24], which in our case guarantees that the energy of the semi-discretized, partitioned problem, mimics the energy of the fully coupled continuous problem. This was crucial for the proof of the existence of a weak solution.

The results of this manuscript effectively show that the partitioned numerical scheme based on the operator splitting approach presented in this paper, converges to a weak solution of the full, 3D nonlinear FSI problem. 


\subsection{Background}

Fluid-structure interaction problems arise in many applications. The widely known examples are aeroelasticity and biofluids. In aeroelasticity, where the structure (wing of an airplane) is much heavier than the fluid (air), it is sometimes of interest to study small vibrations of the structure in which case linear coupling between the fluid and the structure may be sufficient to capture the main features of the solutions. In that case the fluid domain can be considered fixed, and the structure displacement is calculated based on the normal stress exerted by the fluid onto the structure. The fluid affects the motion of the structure, but the structure does not significantly affect the motion of the fluid and so it can be neglected (one-way coupling). In biofluidic applications, such as the interaction between blood flow and cardiovascular tissue where the density of the structure (cardiovascular tissue) is roughly equal to the density of the fluid (blood), the coupling between the fluid and the relatively light structure is highly nonlinear. Both the fluid and the structure are strongly affected by the fluid-structure interaction, and a full coupling based on the contact forces exerted by both the fluid and the structure must be taken into account (two-way coupling). It has recently been shown that the classical "partitioned" time-marching numerical algorithms, which are based on subsequent solutions of the fluid and structure sub-problems, are unconditionally unstable in the blood flow application [8]. The exchange of energy between a moving fluid and a structure is so significant, that a mismatch between the energy of the discretized problem and the energy of the continuous problem causes instabilities in classical "loosely coupled" partitioned schemes. The difficulties associated with the significant energy exchange and the high geometric nonlinearity of the fluidstructure interface are reflected not only in the design of numerical schemes, but also in the theoretical studies of existence and stability of solutions to this class of problems. A comprehensive study of these problems remains to be a challenge due to their strong nonlinearity and multi-physics nature.

\subsection{A brief literature review}

Fluid-structure interaction problems have been extensively studied for the past 20 years by many authors. The field has evolved from first studying FSI between an incompressible, viscous fluid and a rigid structure immersed in a fluid, to considering compliant (elastic/viscoelastic) structures interacting with a fluid. Concerning compliant structures, the coupling between the structure and the fluid was first assumed to take place along a fixed fluid 
domain boundary (linear coupling). This was then extended to FSI problems in which the coupling was evaluated at a deformed fluid-structure interface, giving rise to an additional nonlinearity in the problem (nonlinear coupling).

Well-posedness results in which the structure was assumed to be a rigid body immersed in a fluid, or described by a finite number of modal functions, were studied in $[5,16,19,20,21,25,26,46]$. FSI problems coupling the Navier-Stokes equations with linear elasticity where the coupling was calculated at a fixed fluid domain boundary, were considered in [23], and in $[2,3,35]$ where an additional nonlinear coupling term was added at the interface. A study of well-posedness for FSI problems between an incompressible, viscous fluid and an elastic/viscoelastic structure with nonlinear coupling evaluated at a moving interface started with the result by daVeiga [4], where existence of a strong solution was obtained locally in time for an interaction between a $2 D$ fluid and a $1 D$ viscoelastic string, assuming periodic boundary conditions. This result was extended by Lequeurre in $[38,39]$, where the existence of a unique, local in time, strong solution for any data, and the existence of a global strong solution for small data, was proved in the case when the structure was modeled as a clamped viscoelastic beam. D. Coutand and S. Shkoller proved existence, locally in time, of a unique, regular solution for an interaction between a viscous, incompressible fluid in $3 D$ and a $3 D$ structure, immersed in the fluid, where the structure was modeled by the equations of linear [17], or quasi-linear [18] elasticity. In the case when the structure (solid) is modeled by a linear wave equation, I. Kukavica and A. Tufahha proved the existence, locally in time, of a strong solution, assuming lower regularity for the initial data [33]. A similar result for compressible flows can be found in [34]. A fluid-structure interaction between a viscous, incompressible fluid in $3 D$, and $2 D$ elastic shells was considered in $[11,10]$ where existence, locally in time, of a unique regular solution was proved. All the above mentioned existence results for strong solutions are local in time. We also mention that the works of Shkoller et al., and Kukavica at al. were obtained in the context of Lagrangian coordinates, which were used for both the structure and fluid problems.

In the context of weak solutions, the following results have been obtained. Continuous dependence of weak solutions on initial data for a fluid structure interaction problem with a free boundary type coupling condition was studied in [29]. Existence of a weak solution for a FSI problem between a $3 D$ incompressible, viscous fluid and a $2 D$ viscoelastic plate was considered by Chambolle et al. in [9], while Grandmont improved this result in [28] to hold for a $2 D$ elastic plate. These results were extended to a more general geometry in [37], and then to the case of generalized Newtonian fluids in 
[36], and to a non-Newtonian shear dependent fluid in [40]. In these works existence of a weak solution was proved for as long as the elastic boundary does not touch "the bottom" (rigid) portion of the fluid domain boundary.

Muha and Čanić recently proved the existence of weak solutions to a class of FSI problems modeling the flow of an incompressible, viscous, Newtonian fluid flowing through a 2D cylinder whose lateral wall was modeled by either the linearly viscoelastic, or by the linearly elastic Koiter shell equations [43], assuming nonlinear coupling at the deformed fluid-structure interface. The fluid flow boundary conditions were not periodic, but rather, the flow was driven by the dynamic pressure drop data. The methodology of proof in [43] was based on a semi-discrete, operator splitting Lie scheme, which was used in [30] to design a stable, loosely coupled partitioned numerical scheme, called the kinematically coupled scheme (see also [7]). Ideas based on the Lie operator splitting scheme were also used by Temam in [49] to prove the existence of a solution to the nonlinear Carleman equation. These results were recently extended by Muha and Čanić to a FSI problem with two structural layers [42]. This was a first step toward modeling the FSI between blood flow and arterial walls which are known to be composed of three main layers, separated by thin elastic laminae, each with different mechanical characteristics. In both works, however, a $2 \mathrm{D}$ radially symmetric problem was studied, in which the forcing and the displacement of the structure was assumed to be radially symmetric.

In the present manuscript we extend those results to 3D, and we allow the structure displacement to depend on both the axial and azimuthal variables, without assuming any radial symmetry in the problem.

\section{Model description}

Motivated by modeling blood flow in human arteries, we consider the flow of an incompressible, viscous fluid in a three-dimensional cylindrical domain of reference length $L$, and reference radius $R$, see Figure 1 . We will be assuming that the lateral boundary of the cylinder is deformable and that its location is not known a priori, but is fully coupled to the motion of a viscous, incompressible fluid occupying the fluid domain. Furthermore, it will be assumed that the lateral boundary is a thin, isotropic, homogeneous structure, whose dynamics is modeled by the cylindrical Koiter shell equations. Additionally, for simplicity, we will be assuming that only the radial component of displacement is non-negligible. This is a common assumption in blood flow modeling [44]. In contrast with our previous works, in this manuscript the problem is set in $3 \mathrm{D}$, and the displacement of the structure 
is not assumed to be radially symmetric. Neither the fluid flow, nor the displacement of the lateral boundary of the fluid domain will be required to satisfy the conditions of axial symmetry. As a consequence, the displacement $\eta$ will depend not only on the axial variable $z$ plus time, but also on the azimuthal variable $\theta$. Therefore, the radial displacement from the reference configuration will be denoted by $\eta(t, z, \theta)$. See Figure 1 . Therefore, in this manuscript for the first time, we provide a constructive existence proof based on the Lie operator splitting approach for the full three-dimensional fluid-structure interaction problem between an incompressible-viscous fluid and a linearly elastic Koiter shell, defined on a cylindrical domain which is not necessarily axially symmetric. The operator splitting strategy can be used in the construction of a loosely-coupled computational scheme which we show converges to a weak solution of the underlying FSI problem.

2.0.1. Remark on notation We will be using $(z, x, y)$ to denote the Cartesian coordinates of points in $\mathbb{R}^{3}$, and $(z, r, \theta)$ to denote the corresponding cylindrical coordinates. We will be working with the fluid flow equations written in Cartesian coordinates, while the structure equations will be given in cylindrical coordinates. A function $f$ given in Cartesian coordinates defines a function

$$
\tilde{f}(z, r, \theta)=f(z, x, y)
$$

defined in cylindrical coordinates. Since no confusion is possible, to simplify notation we will omit the superscript ${ }^{\sim}$ and both functions, $f$ and $\tilde{f}$, will be denoted by $f$.

The structural problem: Consider a clamped cylindrical shell of thickness $h$, length $L$, and reference radius of the middle surface equal to $R$. See Figure 1. This reference configuration, which we denote by $\Gamma$, can be defined via the parameterization

$$
\varphi: \omega \rightarrow \mathbb{R}^{3}, \quad \varphi(z, \theta)=(R \cos \theta, R \sin \theta, z)^{t}
$$

where $\omega=(0, L) \times(0,2 \pi)$ and $R>0$. Therefore, the reference configuration is

$$
\Gamma=\left\{\mathbf{x}=(R \cos \theta, R \sin \theta, z) \in \mathbb{R}^{3}: \theta \in(0,2 \pi), z \in(0, L)\right\}
$$

The associated covariant $A_{c}$ and contravariant $A^{c}$ metric tensors of this (undeformed) cylinder are give by:

$$
\mathbf{A}_{c}=\left(\begin{array}{cc}
1 & 0 \\
0 & R^{2}
\end{array}\right), \mathbf{A}^{c}=\left(\begin{array}{cc}
1 & 0 \\
0 & \frac{1}{R^{2}}
\end{array}\right)
$$


and the area element along cylinder $\Gamma$ is $d S=\sqrt{a} d y:=\sqrt{\operatorname{det} A_{c}} d y=R d y$. The corresponding curvature tensor in covariant components is given by

$$
\mathbf{B}_{c}=\left(\begin{array}{cc}
0 & 0 \\
0 & R
\end{array}\right)
$$

Under the action of a force, the Koiter shell is deformed. The displacement from the reference configuration $\Gamma$ of the deformed shell will be denoted by $\boldsymbol{\eta}=\boldsymbol{\eta}(t, z, \theta)=\left(\eta_{z}, \eta_{\theta}, \eta_{r}\right)$. We will be assuming that only the radial component of the displacement is different from zero, and will be denoting that component of the displacement by $\eta(t, z, \theta):=\eta_{r}(t, z, \theta)$, so that $\boldsymbol{\eta}=\eta \mathbf{e}_{r}$, where $\mathbf{e}_{r}=\mathbf{e}_{r}(\theta)=(\cos \theta, \sin \theta, 0)^{t}$ is the unit vector in the radial direction.

The cylindrical Koiter shell is assumed to be clamped at the end points, giving rise to the following boundary conditions:

$$
\eta=\frac{\partial \eta}{\partial \mathbf{n}}=0 \text { on } \partial \omega
$$

Deformation of a given Koiter shell depends on its elastic properties. The elastic properties of our cylindrical Koiter shell are defined by the following elasticity tensor $\mathcal{A}$ :

$$
\mathcal{A} \mathbf{E}=\frac{4 \lambda \mu}{\lambda+2 \mu}\left(\mathbf{A}^{c} \cdot \mathbf{E}\right) \mathbf{A}^{c}+4 \mu \mathbf{A}^{c} \mathbf{E} \mathbf{A}^{c}, \quad \mathbf{E} \in \operatorname{Sym}\left(\mathcal{M}_{2}\right),
$$

where $\mu$ and $\lambda$ are the Lamé coefficients. Using the following relationships between the Lamé constants and the Young's modulus of elasticity $E$ and Poisson ratio $\sigma$ :

$$
\frac{2 \mu \lambda}{\lambda+2 \mu}+2 \mu=4 \mu \frac{\lambda+\mu}{\lambda+2 \mu}=\frac{E}{1-\sigma^{2}}, \quad \frac{2 \mu \lambda}{\lambda+2 \mu}=4 \mu \frac{\lambda+\mu}{\lambda+2 \mu} \frac{1}{2} \frac{\lambda}{\lambda+\mu}=\frac{E}{1-\sigma^{2}} \sigma,
$$

the elasticity tensor $\mathcal{A}$ can also be written as:

$$
\mathcal{A} \mathbf{E}=\frac{2 E \sigma}{1-\sigma^{2}}\left(\mathbf{A}^{c} \cdot \mathbf{E}\right) \mathbf{A}^{c}+\frac{2 E}{1+\sigma} \mathbf{A}^{c} \mathbf{E} \mathbf{A}^{c}, \quad \mathbf{E} \in \operatorname{Sym}\left(\mathcal{M}_{2}\right) .
$$

A Koiter shell can undergo stretching of the middle surface, and flexure (bending). Namely, the Koiter shell model accounts for both the membrane effects (stretching) and shell effects (flexure). Stretching of the middle surface is measured by the change of metric tensor, while flexure is measured by the change of curvature tensor. By assuming only the radial component of displacement $\eta=\eta(t, r, \theta)$ to be different from zero, the linearized change 
of metric tensor $\boldsymbol{\gamma}$, and the linearized change of curvature tensor $\boldsymbol{\rho}$, are given by the following:

$$
\gamma(\eta)=\left(\begin{array}{cc}
0 & 0 \\
0 & R \eta
\end{array}\right), \quad \boldsymbol{\rho}(\eta)=\left(\begin{array}{cc}
-\partial_{z}^{2} \eta & -\partial_{z \theta}^{2} \eta \\
-\partial_{z \theta}^{2} \eta & -\partial_{\theta}^{2} \eta+\eta
\end{array}\right) .
$$

With the corresponding change of metric and change of curvature tensors we can now formally write the corresponding elastic energy of the deformed shell $[14,12,13,32]$ :

$$
E_{e l}(\eta)=\frac{h}{4} \int_{\omega} \mathcal{A} \gamma(\eta): \gamma(\eta) R+\frac{h^{3}}{48} \int_{\omega} \mathcal{A} \boldsymbol{\rho}(\eta): \boldsymbol{\rho}(\eta) R,
$$

where $h$ is the thickness of the shell, and : denotes the scalar product

$$
\mathbf{A}: \mathbf{B}:=\operatorname{Tr}\left(\mathbf{A B}^{T}\right) \mathbf{A}, \mathbf{B} \in \mathbf{M}_{2}(\mathbb{R}) \cong \mathbb{R}^{4} .
$$

Given a force $\mathbf{f}=f \mathbf{e}_{r}$, with surface density $f$ (the radial component), the loaded shell deforms under the applied force, and the corresponding displacement $\eta$ is a solution to the following elastodyamics problem for the cylindrical linearly elastic Koiter shell, written in weak form: Find $\eta \in H_{0}^{2}(\omega)$ such that

$$
\begin{aligned}
& \rho_{K} h \int_{\omega} \partial_{t}^{2} \eta \psi R+\frac{h}{2} \int_{\omega} \mathcal{A} \gamma(\eta): \gamma(\psi) R+\frac{h^{3}}{24} \int_{\omega} \mathcal{A} \boldsymbol{\rho}(\eta): \boldsymbol{\rho}(\psi) R \\
& \quad=\int_{\omega} f \psi R, \forall \psi \in H_{0}^{2}(\omega) .
\end{aligned}
$$

We define an elastic operator $\mathcal{L}$ :

$$
\int_{\omega} \mathcal{L} \eta \psi=\frac{h}{2} \int_{\omega} \mathcal{A} \gamma(\eta): \gamma(\psi) R+\frac{h^{3}}{24} \int_{\omega} \mathcal{A} \boldsymbol{\rho}(\eta): \boldsymbol{\rho}(\psi) R, \forall \psi \in H_{0}^{2}(\omega),
$$

so that the above weak formulation can be written as

$$
\rho_{K} h \int_{\omega} \partial_{t}^{2} \eta \psi R+\int_{\omega} \mathcal{L} \eta \psi=\int_{\omega} f \psi R, \forall \psi \in H_{0}^{2}(\omega) .
$$

A calculation shows that the operator $\mathcal{L}$ written in differential form reads: (2.8)

$$
\begin{aligned}
\mathcal{L} \eta & =\frac{h^{3} \mu}{3 R^{3}(\lambda+2 \mu)}\left((\lambda+\mu) \partial_{\theta}^{4} \eta+R^{4}(\lambda+\mu) \partial_{z}^{4} \eta+2 R^{2}(\lambda+\mu) \partial_{z}^{2} \partial_{\theta}^{2} \eta\right. \\
& \left.-R^{2} \lambda \partial_{z}^{2} \eta-2(\lambda+\mu) \partial_{\theta}^{2} \eta+(\lambda+\mu) \eta\right)+\frac{4 h}{R} \frac{(\lambda+\mu) \mu}{\lambda+2 \mu} \eta .
\end{aligned}
$$


In terms of the Youngs modulus of elasticity, and the Poisson ratio, operator $\mathcal{L}$ can be written as:

$$
\begin{aligned}
\mathcal{L} \eta & =\frac{h^{3} E}{12 R^{4}\left(1-\sigma^{2}\right)}\left(\partial_{\theta}^{4} \eta+R^{4} \partial_{z}^{4} \eta+2 R^{2} \partial_{z}^{2} \partial_{\theta}^{2} \eta-2 \partial_{\theta}^{2} \eta+\eta\right) \\
& +\frac{h^{3} E \sigma}{6 R^{2}\left(1-\sigma^{2}\right)} \partial_{z}^{2} \eta+\frac{h E}{R^{2}\left(1-\sigma^{2}\right)} \eta .
\end{aligned}
$$

The fluid problem: The fluid domain, which depends on time and is not known a priori, will be denoted by

$$
\Omega_{\eta}(t)=\left\{(z, x, y) \in \mathbb{R}^{3}: \sqrt{x^{2}+y^{2}}<R+\eta(t, z, \theta), z \in(0, L)\right\},
$$

and the corresponding lateral boundary by

$$
\Gamma_{\eta}(t)=\left\{(z, x, y) \in \mathbb{R}^{3}: \sqrt{x^{2}+y^{2}}=R+\eta(t, z, \theta), z \in(0, L)\right\} .
$$

The inlet and outlet sections of the fluid domain boundary will be denoted by $\Gamma_{\text {in }}=\{0\} \times(0, R), \Gamma_{\text {out }}=\{L\} \times(0, R)$. See Figure 1 .

We are interested in studying a dynamic pressure-driven flow through $\Omega_{\eta}(t)$ of an incompressible, viscous fluid modeled by the Navier-Stokes equations which are given in Cartesian coordinates:

$$
\left.\begin{array}{rl}
\rho_{f}\left(\partial_{t} \mathbf{u}+\mathbf{u} \cdot \nabla \mathbf{u}\right) & =\nabla \cdot \sigma, \\
\nabla \cdot \mathbf{u} & =0,
\end{array}\right\} \text { in } \Omega_{\eta}(t), t \in(0, T),
$$

where $\rho_{f}$ denotes the fluid density, $\mathbf{u}$ fluid velocity, $p$ fluid pressure,

$$
\sigma=-p \mathbf{I}+2 \mu_{F} \mathbf{D}(\mathbf{u})
$$

is the fluid Cauchy stress tensor, $\mu_{F}$ is the kinematic viscosity coefficient, and $\mathbf{D}(\mathbf{u})=\frac{1}{2}\left(\nabla \mathbf{u}+\nabla^{t} \mathbf{u}\right)$ is the symmetrized gradient of $\mathbf{u}$.

At the inlet and outlet boundaries we prescribe zero tangential velocity and dynamic pressure $p+\frac{\rho_{f}}{2}|u|^{2}$ (see e.g. [15]):

$$
\left.\begin{array}{rl}
p+\frac{\rho_{f}}{2}|u|^{2} & =P_{\text {in } / \text { out }}(t), \\
\mathbf{u} \times \mathbf{e}_{z} & =0,
\end{array}\right\} \quad \text { on } \Gamma_{\text {in } / \text { out }},
$$

where $P_{\text {in } / \text { out }} \in L_{\text {loc }}^{2}(0, \infty)$ are given. Therefore the fluid flow is driven by a prescribed dynamic pressure drop, and the flow enters and leaves the fluid domain orthogonally to the inlet and outlet boundary. 
The coupling between the fluid and structure is defined by two sets of boundary conditions satisfied at the lateral boundary $\Gamma_{\eta}(t)$. They are the kinematic and dynamic lateral boundary conditions describing continuity of velocity (the no-slip condition), and balance of contact forces (i.e., the Second Newton's Law of motion). Written in the Lagrangian framework, with $(z, \theta) \in \omega$, and $t \in(0, T)$, they read:

\section{- The kinematic condition:}

$$
\partial_{t} \eta(t, z, \theta) \mathbf{e}_{r}(\theta)=\boldsymbol{u}(t, z, R+\eta(t, z, \theta), \theta),
$$

where $\mathbf{e}_{r}(\theta)=(\cos \theta, \sin \theta, 0)^{t}$ is the unit vector in the radial direction.

- The dynamic condition:

$$
\rho_{K} h \partial_{t}^{2} \eta+\mathcal{L} \eta=-\left.J(t, z, \theta)(\sigma \mathbf{n})\right|_{(t, z, R+\eta(t, z, \theta))} \cdot \mathbf{e}_{r}(\theta)
$$

where $\mathcal{L}$ is defined by (2.8), or equivalently by (2.9), and

$$
J(t, z, \theta)=\sqrt{\left(1+\partial_{z} \eta(t, z, \theta)^{2}\right)(R+\eta(t, z, \theta))^{2}+\partial_{\theta} \eta(t, z, \theta)^{2}}
$$

denotes the Jacobian of the composition of the transformation from Eulerian to Lagrangian coordinates and the transformation from cylindrical to Cartesian coordinates. tions:

System (2.10)-(2.13) is supplemented with the following initial condi-

$$
\mathbf{u}(0, .)=\mathbf{u}_{0}, \eta(0, .)=\eta_{0}, \partial_{t} \eta(0, .)=v_{0}
$$

Additionally, we will be assuming that the initial data satisfies the following compatibility conditions:

$$
\begin{aligned}
& \mathbf{u}_{0}\left(z, R+\eta_{0}(z), \theta\right) \cdot \mathbf{n}(z, \theta)= v_{0}(z, \theta) \mathbf{e}_{r}(\theta) \cdot \mathbf{n}(z, \theta), \quad z \in(0, L), \theta \in(0,2 \pi), \\
& \eta_{0}=0, \quad \text { on } \partial \omega, \\
& R+\eta_{0}(z, \theta)>0, \quad z \in[0, L], \theta \in(0,2 \pi) .
\end{aligned}
$$

Notice that the last condition requires that the initial displacement is such that the fluid domain has radius strictly greater than zero (i.e., the lateral boundary never collapses). This is an important condition which will be used at several places throughout this manuscript.

In summary, we study the following fluid-structure interaction problem:

Problem 2.1. Find $\mathbf{u}=\left(u_{z}(t, z, x, y), u_{x}(t, z, x, y), u_{y}(t, z, x, y)\right), p(t, z$, $x, y)$, and $\eta(t, z, \theta)$ such that 


$$
\begin{aligned}
& \left.\begin{array}{rl}
\rho_{f}\left(\partial_{t} \mathbf{u}+(\mathbf{u} \cdot \nabla) \mathbf{u}\right) & =\nabla \cdot \sigma \\
\nabla \cdot \mathbf{u} & =0
\end{array}\right\} \text { in } \Omega_{\eta}(t), t \in(0, T), \\
& \left.\begin{array}{rl}
\left.\mathbf{u}\right|_{R+\eta} & =\partial_{t} \eta \mathbf{e}_{r}, \\
\rho_{K} h \partial_{t}^{2} \eta+\mathcal{L} \eta & =-J \sigma \mathbf{n} \cdot \mathbf{e}_{r},
\end{array}\right\} \text { on }(0, T) \times \omega, \\
& \left.\begin{array}{rl}
p+\frac{\rho_{f}}{2}|u|^{2} & =P_{\text {in } / \text { out }}(t), \\
\mathbf{u} \times \mathbf{e}_{z} & =0,
\end{array}\right\} \text { on }(0, T) \times \Gamma_{\text {in } / \text { out }}, \\
& \left.\begin{array}{rl}
\mathbf{u}(0, .) & =\mathbf{u}_{0}, \\
\eta(0, .) & =\eta_{0}, \\
\partial_{t} \eta(0, .) & =v_{0} .
\end{array}\right\} \text { at } t=0 .
\end{aligned}
$$

This is a nonlinear, moving-boundary problem in 3D, which captures the full, two-way fluid-structure interaction coupling. The nonlinearity in the problem is represented by the quadratic term in the fluid equations, and by the nonlinear coupling between the fluid and structure defined at the lateral boundary $\Gamma_{\eta}(t)$, which is one of the unknowns in the problem.

\subsection{Energy inequality}

Assuming sufficient regularity, we formally derive an energy inequality for the coupled FSI problem summarized above in Problem 2.1. To simplify notation, we introduce the following energy norms defined by the membrane and flexural effects of the linearly elastic Koiter shell:

$$
\|f\|_{\gamma}:=\int_{\omega} \mathcal{A} \gamma(f): \gamma(f) R, \quad\|f\|_{\sigma}:=\int_{\omega} \mathcal{A} \boldsymbol{\sigma}(f): \boldsymbol{\sigma}(f) R .
$$

Notice that norm $\|\cdot\|_{\gamma}$ is equivalent to the standard $L^{2}(\omega)$ norm, and that norm $\|\cdot\|_{\sigma}$ is equivalent to the standard $H_{0}^{2}(\omega)$ norm.

Proposition 2.1. Assuming sufficient regularity, solutions of Problem 2.1 satisfy the following energy estimate:

$$
\frac{d}{d t}\left(E_{k i n}(t)+E_{\text {el }}(t)\right)+D(t) \leq C\left(P_{\text {in }}(t), P_{\text {out }}(t)\right),
$$

where

$$
\begin{aligned}
& E_{k i n}(t):= \frac{1}{2}\left(\rho_{f}\|\mathbf{u}\|_{L^{2}\left(\Omega_{F}(t)\right)}^{2}+\rho_{K} h\left\|\partial_{t} \eta\right\|_{L^{2}(\Gamma)}^{2}\right), \\
& E_{e l}(t):=\frac{h}{4}\|\eta\|_{\gamma}+\frac{h^{3}}{48}\|\eta\|_{\sigma}
\end{aligned}
$$


denote the kinetic and elastic energy of the coupled problem, respectively, and the term $D(t)$ captures viscous dissipation in the fluid:

$$
D(t):=\mu_{F}\|\mathbf{D}(\mathbf{u})\|_{L^{2}\left(\Omega_{F}(t)\right)}^{2} .
$$

The constant $C\left(P_{\text {in }}(t), P_{\text {out }}(t)\right)$ depends only on the inlet and outlet pressure data, which are both functions of time.

The proof of inequality (2.21) is standard (see, e.g., [43]), so we omit it here.

Later in the manuscript, it will be rigorously shown that the weak solutions constructed in this manuscript, satisfy the above energy estimate. Namely, the goal of this manuscript is to show the following existence result for weak solutions of Problem 2.1:

Main result. Let the model parameters satisfy $\rho_{f}, \rho_{K}, \mu_{F}, h, \mu, \lambda>0$. Suppose that the initial data $v_{0} \in L^{2}(\omega), \mathbf{u}_{0} \in L^{2}\left(\Omega_{\eta_{0}}\right)$, and $\eta_{0} \in H_{0}^{2}(\omega)$ are such that the initial tube radius is strictly greater than zero: $\left(R+\eta_{0}(z, \theta)\right)>$ $0,(z, \theta) \in \omega$. Furthermore, let $P_{\text {in }}, P_{\text {out }} \in L_{\text {loc }}^{2}(0, \infty)$.

Then there exist a $T>0$ and a weak solution $(\mathbf{u}, \eta)$ of Problem 3.1 on $(0, T)$ in the sense of Definition 3.1 below, that satisfy the following energy estimate:

$$
E(t)+\int_{0}^{t} D(\tau) d \tau \leq E_{0}+C\left(\left\|P_{\text {in }}\right\|_{L^{2}(0, t)}^{2}+\left\|P_{\text {out }}\right\|_{L^{2}(0, t)}^{2}\right), \quad t \in[0, T],
$$

where $C$ depends only on the coefficients in the problem, $E_{0}$ is the kinetic energy of the initial data, and $E(t)$ and $D(t)$ are given by

$$
\begin{aligned}
& E(t)=\frac{\rho_{f}}{2}\|\mathbf{u}\|_{L^{2}\left(\Omega_{\eta}(t)\right)}^{2}+\frac{\rho_{K} h}{2}\left\|\partial_{t} \eta\right\|_{L^{2}(\omega)}^{2}+\frac{h}{4}\|\eta\|_{\gamma}^{2}+\frac{h^{3}}{48}\|\eta\|_{\sigma}^{2}, \\
& D(t)=\mu_{F}\|\mathbf{D}(\mathbf{u})\|_{L^{2}\left(\Omega_{\eta}(t)\right)}^{2},
\end{aligned}
$$

where

$$
\|f\|_{\gamma}:=\int_{\omega} \mathcal{A} \gamma(f): \gamma(f) R, \quad\|f\|_{\sigma}:=\int_{\omega} \mathcal{A} \boldsymbol{\sigma}(f): \sigma(f) R,
$$

are the energy norms defined by the membrane and flexural effects of the linearly elastic Koiter shell, where the elasticity operator $\mathcal{A}$ is defined in (2.2). They are equivalent to the standard $L^{2}(\omega)$ and $H_{0}^{2}(\omega)$ norms, respectively. 


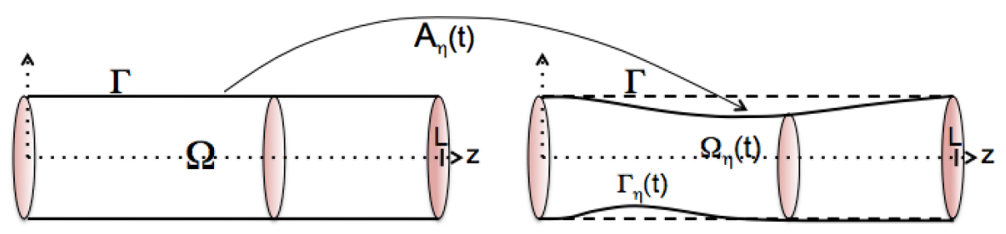

Figure 2: ALE mapping.

Furthermore, one of the following is true: either

(1) $T=\infty$, or

(2) $\lim _{t \rightarrow T} \min _{z \in[0, L]}(R+\eta(z))=0$.

\section{Weak solution}

\subsection{ALE mapping}

To prove the existence of a weak solution to Problem 2.1 it is convenient to map Problem 2.1 onto a fixed domain $\Omega$. In our approach we choose $\Omega$ to be a cylinder of radius 1 and length $L$

$$
\Omega=\left\{(z, x, y): z \in(0, L), x^{2}+y^{2}<1\right\} .
$$

We follow the approach typical of numerical methods for fluid-structure interaction problems and map our fluid domain $\Omega(t)$ onto $\Omega$ by using an Arbitrary Lagrangian-Eulerian (ALE) mapping [7, 30, 22, 45]. We remark here that in our problem it is not convenient to use the Lagrangian formulation of the fluid sub-problem, as is done in e.g., [18, 11, 33], since, in our problem, the fluid domain consists of a fixed, control volume of a cylinder, which does not follow Largangian flow.

We begin by defining a family of ALE mappings $A_{\eta}$ parameterized by $\eta$ : $(3.1)$

$A_{\eta}(t): \Omega \rightarrow \Omega_{\eta}(t), \quad A_{\eta}(t)(\tilde{z}, \tilde{r}, \tilde{\theta}):=\left(\begin{array}{c}\tilde{z} \\ (R+\underset{\tilde{\theta}}{t}, \tilde{z}, \tilde{\theta})) \tilde{r}\end{array}\right), \quad(\tilde{z}, \tilde{r}, \tilde{\theta}) \in \Omega$,

where $(\tilde{z}, \tilde{r}, \tilde{\theta})$ denote the cylindrical coordinates in the reference domain $\Omega$. See Figure 2. Since we work with the Navier-Stokes equations written in Cartesian coordinates, it is useful to write an explicit form of the ALE 
mapping $A_{\eta}$ in the Cartesian coordinates as well:

$$
A_{\eta}(t)(\tilde{z}, \tilde{x}, \tilde{y}):=\left(\begin{array}{c}
\tilde{z} \\
(R+\eta(t, \tilde{z}, \tilde{\theta})) \tilde{x} \\
(R+\eta(t, \tilde{z}, \tilde{\theta})) \tilde{y}
\end{array}\right), \quad(\tilde{z}, \tilde{x}, \tilde{y}) \in \Omega .
$$

Mapping $A_{\eta}(t)$ is a bijection, and its Jacobian is given by

$$
\left|\operatorname{det} \nabla A_{\eta}(t)\right|=(R+\eta(t, \tilde{z}, \tilde{\theta}))^{2} .
$$

Composite functions with the ALE mapping will be denoted by

$$
\mathbf{u}^{\eta}(t, .)=\mathbf{u}(t, .) \circ A_{\eta}(t) \text { and } p^{\eta}(t, .)=p(t, .) \circ A_{\eta}(t)
$$

The derivatives of composite functions satisfy:

$$
\nabla \mathbf{u}=\nabla \mathbf{u}^{\eta}\left(\nabla A_{\eta}\right)^{-1}=: \nabla^{\eta} \mathbf{u}^{\eta}, \quad \partial_{t} \mathbf{u}=\partial_{t} \mathbf{u}^{\eta}-\left(\mathbf{w}^{\eta} \cdot \nabla^{\eta}\right) \mathbf{u}^{\eta},
$$

where the ALE domain velocity, $\mathbf{w}^{\eta}$, is given by:

$$
\mathbf{w}^{\eta}=\partial_{t} \eta\left(\begin{array}{c}
0 \\
\tilde{x} \\
\tilde{y}
\end{array}\right)
$$

The following notation will also be useful:

$$
\sigma^{\eta}=-p^{\eta} \mathbf{I}+2 \mu \mathbf{D}^{\eta}\left(\mathbf{u}^{\eta}\right), \quad \mathbf{D}^{\eta}\left(\mathbf{u}^{\eta}\right)=\frac{1}{2}\left(\nabla^{\eta} \mathbf{u}^{\eta}+\left(\nabla^{\eta}\right)^{\tau} \mathbf{u}^{\eta}\right) .
$$

We are now ready to rewrite Problem 2.1 in the ALE formulation. However, before we do that, we will make one more important step in our strategy to prove the existence of a weak solution to Problem 2.1. Namely, we would like to "solve" the coupled FSI problem by approximating the problem using time-discretization via operator splitting, and then prove that the solution to the semi-discrete problem converges to a weak solution of the continuous problem, as the time-discretization step tends to zero. To perform time discretization via operator splitting, which will be described in the next section, we need to write our FSI problem as a first-order system in time. This will be done by replacing the second-order time-derivative of $\eta$, with the first-order time-derivative of the structure velocity. To do this, we further notice that in the coupled FSI problem, the kinematic coupling condition (2.12) implies that the structure velocity is equal to the normal trace of the 
fluid velocity on $\Gamma_{\eta}(t)$. Thus, we will introduce a new variable, $v$, to denote this trace, and will replace $\partial_{t} \eta$ by $v$ everywhere in the structure equation. This has deep consequences both for the existence proof presented in this manuscript, as well as for the proof of stability of the underlying numerical scheme, presented in [50], as it enforces the kinematic coupling condition implicitly in all the steps of the scheme.

Thus, Problem 2.1 can be reformulated in the ALE framework, on the reference domain $\Omega$, and written as a first-order system in time, in the following way:

Problem 3.1. Find $\mathbf{u}^{\eta}(t, \tilde{z}, \tilde{x}, \tilde{y}), p^{\eta}(t, \tilde{z}, \tilde{x}, \tilde{y}), \eta(t, \tilde{z}, \tilde{\theta})$, and $v(t, \tilde{z}, \tilde{\theta})$ such that

$$
\begin{aligned}
& \left.\begin{array}{rl}
\rho_{f}\left(\partial_{t} \mathbf{u}^{\eta}+\left(\left(\mathbf{u}^{\eta}-\mathbf{w}^{\eta}\right) \cdot \nabla^{\eta}\right) \mathbf{u}^{\eta}\right) & =\nabla^{\eta} \cdot \sigma^{\eta}, \\
\nabla^{\eta} \cdot \mathbf{u}^{\eta} & =0,
\end{array}\right\} \text { in }(0, T) \times \Omega, \\
& \left.\begin{array}{rl}
p+\frac{\rho_{f}}{2}\left|u^{\eta}\right|^{2} & =P_{\text {in/out }}(t), \\
\mathbf{u}^{\eta} \times \mathbf{e}_{z} & =0,
\end{array}\right\} \text { on }(0, T) \times \Gamma_{\text {in } / \text { out }}, \\
& \left.\begin{array}{rl}
\mathbf{u}^{\eta} & =v \mathbf{e}_{r}, \\
\partial_{t} \eta & =v, \\
\rho_{K} h \partial_{t} v+\mathcal{L} \eta & =-J \sigma^{\eta} \mathbf{n} \cdot \mathbf{e}_{r}
\end{array}\right\} \text { on }(0, T) \times(0, L), \\
& \mathbf{u}^{\eta}(0, .)=\mathbf{u}_{0}, \eta(0, .)=\eta_{0}, v(0, .)=v_{0}, \quad \text { at } \quad t=0 .
\end{aligned}
$$

To simplify notation, in the text that follows, will drop the superscript $\eta$ in $\mathbf{u}^{\eta}$ whenever there is no chance of confusion.

\subsection{Weak formulation}

To define weak solutions of the moving-boundary problem (3.6)-(3.9) defined on a fixed domain, we introduce the following notation and function spaces.

For the fluid velocity we would like to work with the classical function space associated with weak solutions of the Navier-Stokes equations. This, however, requires some additional consideration. Namely, since the fluid domain is also an unknown in the problem, we cannot assume a priori any smoothness that is not consistent with the energy estimates, and so the fluid domain boundary may not even be Lipschitz. Indeed, from the energy inequality (2.21) we only have $\eta \in H^{2}(\omega)$, and from Sobolev embeddings, by taking into account that we are working in $\mathbb{R}^{3}$, we get that $\eta \in C^{0, \mu}(\omega)$, $\mu<1$. Therefore, the energy estimates imply that $\Omega_{\eta}(t)$ is not necessarily a Lipschitz domain. However, $\Omega_{\eta}(t)$ is locally a sub-graph of a Hölder 
continuous function. In that case one can define the "Lagrangian" trace

$$
\begin{aligned}
\gamma_{\Gamma(t)} & : C^{1}\left(\overline{\Omega_{\eta}(t)}\right) \rightarrow C(\omega), \\
\gamma_{\Gamma(t)} & : v \mapsto v(t, \tilde{z}, 1+\eta(t, \tilde{z}, \tilde{\theta}), \tilde{\theta}) .
\end{aligned}
$$

It was shown in $[9,28,41]$ that the trace operator $\gamma_{\Gamma(t)}$ can be extended by continuity to a linear operator from $H^{1}\left(\Omega_{\eta}(t)\right)$ to $H^{s}(\omega), 0 \leq s<\frac{1}{2}$. For a precise statement of the results about "Lagrangian" trace see [41]. Now, we can define the velocity solution space defined on the moving domain in the following way:

$$
\begin{aligned}
V_{F}(t)= & \left\{\mathbf{u}=\left(u_{z}, u_{x}, u_{y}\right) \in C^{1}\left(\overline{\Omega_{\eta}(t)}\right)^{3}: \nabla \cdot \mathbf{u}=0,\right. \\
& \left.\mathbf{u} \times \mathbf{e}_{r}=0 \text { on } \Gamma(t), \mathbf{u} \times \mathbf{e}_{z}=0 \text { on } \Gamma_{\text {in } / \text { out }}\right\}, \\
\mathcal{V}_{F}(t)= & \bar{V}_{F}(t) H^{1}\left(\Omega_{\eta}(t)\right)
\end{aligned}
$$

Using the fact that $\Omega_{\eta}(t)$ is locally a sub-graph of a Hölder continuous function we can get the following characterization of the velocity solution space $\mathcal{V}_{F}(t)($ see $[9,28])$ :

$$
\begin{aligned}
\mathcal{V}_{F}(t)= & \left\{\mathbf{u}=\left(u_{z}, u_{x}, u_{y}\right) \in H^{1}\left(\Omega_{\eta}(t)\right)^{3}: \nabla \cdot \mathbf{u}=0,\right. \\
& \left.\mathbf{u} \times \mathbf{e}_{r}=0 \text { on } \Gamma(t), \mathbf{u} \times \mathbf{e}_{z}=0 \text { on } \Gamma_{\text {in } / \text { out }}\right\} .
\end{aligned}
$$

Before defining the fluid velocity space defined on a fixed, reference domain $\Omega$, it is important to point out that the transformed fluid velocity $\mathbf{u}^{\eta}$ is not divergence-free anymore. Rather, it satisfies the transformed divergencefree condition $\nabla^{\eta} \cdot \mathbf{u}^{\eta}=0$. Furthermore, since $\eta$ is not Lipschitz, the ALE mapping is not necessarily a Lipschitz function either, and, as a result, $\mathbf{u}^{\eta}$ is not necessarily in $H^{1}(\Omega)$. Therefore, we need to redefine the function spaces for the fluid velocity by introducing

$$
\mathcal{V}_{F}^{\eta}=\left\{\mathbf{u}^{\eta}: \mathbf{u} \in \mathcal{V}_{F}(t)\right\}
$$

where $\mathbf{u}^{\eta}$ is defined in (3.4). Under the assumption $R+\eta(t, \tilde{z}, \tilde{\theta})>0, \tilde{z} \in$ $[0, L]$, we can define a scalar product on $\mathcal{V}_{F}^{\eta}$ in the following way:

$$
\begin{array}{r}
\left(\mathbf{u}^{\eta}, \mathbf{v}^{\eta}\right)_{\mathcal{V}_{F}^{\eta}}=\int_{\Omega}(R+\eta)^{2}\left(\mathbf{u}^{\eta} \cdot \mathbf{v}^{\eta}+\nabla^{\eta} \mathbf{u}^{\eta}: \nabla^{\eta} \mathbf{v}^{\eta}\right) \\
=\int_{\Omega_{\eta}(t)} \mathbf{u} \cdot \mathbf{v}+\nabla \mathbf{u}: \nabla \mathbf{v}=(\mathbf{u}, \mathbf{v})_{H^{1}\left(\Omega_{\eta}(t)\right)}
\end{array}
$$


Therefore, $\mathbf{u} \mapsto \mathbf{u}^{\eta}$ is an isometric isomorphism between $\mathcal{V}_{F}(t)$ and $\mathcal{V}_{F}^{\eta}$, and so $\mathcal{V}_{F}^{\eta}$ is also a Hilbert space.

The function space associated with the Koiter shell equations is standard:

$$
\mathcal{V}_{K}=H_{0}^{2}(\omega) .
$$

From this point on we will be working with the FSI problem mapped via the ALE mapping onto the fixed, reference domain $\Omega$. Motivated by the energy inequality we define the corresponding evolution spaces for the FSI problem defined on $\Omega$ :

$$
\begin{array}{r}
\mathcal{W}_{F}^{\eta}(0, T)=L^{\infty}\left(0, T ; L^{2}(\Omega)\right) \cap L^{2}\left(0, T ; \mathcal{V}_{F}^{\eta}\right), \\
\mathcal{W}_{K}(0, T)=W^{1, \infty}\left(0, T ; L^{2}(\omega)\right) \cap L^{2}\left(0, T ; H_{0}^{2}(\omega)\right),
\end{array}
$$

The corresponding solution and test spaces are defined by:

$$
\begin{array}{r}
\mathcal{W}^{\eta}(0, T)=\left\{(\mathbf{u}, \eta) \in \mathcal{W}_{F}^{\eta}(0, T) \times \mathcal{W}_{K}(0, T): \mathbf{u}_{\mid r=1}=\partial_{t} \eta \mathbf{e}_{r},\right\} \\
\mathcal{Q}^{\eta}(0, T)=\left\{(\mathbf{q}, \psi) \in C_{c}^{1}\left([0, T) ; \mathcal{V}_{F}^{\eta} \times \mathcal{V}_{K}\right): \mathbf{q}_{\mid r=1}=\psi \mathbf{e}_{r}\right\}
\end{array}
$$

We will be using $b^{\eta}$ to denote the following trilinear form corresponding to the (symmetrized) nonlinear advection term in the Navier-Stokes equations in the fixed, reference domain:

$b^{\eta}(\mathbf{u}, \mathbf{u}, \mathbf{q}):=\frac{1}{2} \int_{\Omega}(R+\eta)^{2}\left(\left(\mathbf{u}-\mathbf{w}^{\eta}\right) \cdot \nabla^{\eta}\right) \mathbf{u} \cdot \mathbf{q}-\frac{1}{2} \int_{\Omega}(R+\eta)^{2}\left(\left(\mathbf{u}-\mathbf{w}^{\eta}\right) \cdot \nabla^{\eta}\right) \mathbf{q} \cdot \mathbf{u}$,

Finally, we define a linear functional which associates the inlet and outlet dynamic pressure boundary data to a test function $\mathbf{v}$ in the following way:

$$
\langle F(t), \mathbf{v}\rangle_{\Gamma_{\text {in } / \text { out }}}=P_{\text {in }}(t) \int_{\Gamma_{\text {in }}} v_{z}-P_{\text {out }}(t) \int_{\Gamma_{\text {out }}} v_{z} .
$$

Definition 3.1. We say that $(\mathbf{u}, \eta) \in \mathcal{W}^{\eta}(0, T)$ is a weak solution of problem (3.6)-(3.9) defined on the reference domain $\Omega$, if for every $(\mathbf{q}, \psi) \in \mathcal{Q}^{\eta}(0, T)$ the following equality holds:

$$
\begin{gathered}
-\rho_{f}\left(\int_{0}^{T} \int_{\Omega}(R+\eta)^{2} \mathbf{u} \cdot \partial_{t} \mathbf{q}+\int_{0}^{T} b^{\eta}(\mathbf{u}, \mathbf{u}, \mathbf{q})\right) \\
+2 \mu_{F} \int_{0}^{T} \int_{\Omega}(R+\eta)^{2} \mathbf{D}^{\eta}(\mathbf{u}): \mathbf{D}^{\eta}(\mathbf{q})
\end{gathered}
$$




$$
\begin{aligned}
& -\rho_{f} \int_{0}^{T} \int_{\Omega}(R+\eta)\left(\partial_{t} \eta\right) \mathbf{u} \cdot \mathbf{q}-R \rho_{K} h \int_{0}^{T} \int_{\omega} \partial_{t} \eta \partial_{t} \psi \\
& +\frac{R h}{2} \int_{0}^{T} \int_{\omega} \mathcal{A} \gamma(\eta): \gamma(\psi)+\frac{R h^{3}}{24} \int_{0}^{T} \int_{\omega} \mathcal{A} \boldsymbol{\rho}(\eta): \boldsymbol{\rho}(\psi) \\
& =\int_{0}^{T}\langle F(t), \mathbf{q}\rangle_{\Gamma_{\text {in } / \text { out }}}+\int_{\Omega_{\eta}}\left(R+\eta_{0}\right)^{2} \mathbf{u}_{0} \cdot \mathbf{q}(0)+\int_{\omega} v_{0} \psi(0) .
\end{aligned}
$$

The weak formulation is obtained in the standard way by multiplying the PDE by a test function and integrating by parts. The only term that is not standard is the third term on the left hand-side of (3.18). This term is obtained from integration by parts of one half of the nonlinear advection term, and it corresponds to the integral $-\rho_{f} \int_{0}^{T} \int_{\Omega}(R+\eta)^{2}\left(\nabla^{\eta} \cdot \mathbf{w}^{\eta}\right) \mathbf{u} \cdot \mathbf{q}$. We calculate

$$
\begin{aligned}
\nabla^{\eta} \cdot \mathbf{w}^{\eta} & =\nabla \cdot \mathbf{w}=\partial_{x}\left(\partial_{t} \eta \frac{x}{R+\eta}\right)+\partial_{y}\left(\partial_{t} \eta \frac{y}{R+\eta}\right) \\
& =x \partial_{x} \frac{\partial_{t} \eta}{R+\eta}+y \partial_{y} \frac{\partial_{t} \eta}{R+\eta}+2 \frac{\partial_{t} \eta}{R+\eta}
\end{aligned}
$$

Now we notice that for any given function $f$ that depends only on $t, z$ and $\theta$, for example $f=\partial_{t} \eta /(R+\eta)$, the following holds

$$
x \partial_{x} f+y \partial_{y} f=\partial_{\theta} f \frac{-x y}{x^{2}+y^{2}}+\partial_{\theta} f \frac{x y}{x^{2}+y^{2}}=0 .
$$

Thus, we obtain

$$
\nabla^{\eta} \cdot \mathbf{w}^{\eta}=\nabla \cdot \mathbf{w}=x \partial_{x} \frac{\partial_{t} \eta}{R+\eta}+y \partial_{y} \frac{\partial_{t} \eta}{R+\eta}+2 \frac{\partial_{t} \eta}{R+\eta}=2 \frac{\partial_{t} \eta}{R+\eta} .
$$

Plugging this expression for $\nabla^{\eta} \cdot \mathbf{w}^{\eta}$ into $-\rho_{f} \int_{0}^{T} \int_{\Omega}(R+\eta)^{2}\left(\nabla^{\eta} \cdot \mathbf{w}^{\eta}\right) \mathbf{u} \cdot \mathbf{q}$ gives the third term in equation (3.18).

\section{Approximate solutions}

\subsection{Lie splitting}

To define approximate solutions we use the time discretization via Lie splitting, also known as the Marchuk-Yanenko splitting scheme. The splitting can be summarized as follows. Let $N \in \mathbb{N}, \Delta t=T / N$ and $t_{n}=n \Delta t$. Consider 
the following initial-value problem:

$$
\frac{d \phi}{d t}+A \phi=0 \quad \text { in }(0, T), \quad \phi(0)=\phi_{0},
$$

where $A$ is an operator defined on a Hilbert space, and $A$ can be written as $A=A_{1}+A_{2}$. Set $\phi^{0}=\phi_{0}$, and, for $n=0, \ldots, N-1$ and $i=1,2$, compute $\phi^{n+\frac{i}{2}}$ by solving

$$
\left.\begin{array}{rl}
\frac{d}{d t} \phi_{i}+A_{i} \phi_{i} & =0 \\
\phi_{i}\left(t_{n}\right) & =\phi^{n+\frac{i-1}{2}}
\end{array}\right\} \quad \text { in }\left(t_{n}, t_{n+1}\right)
$$

and then set $\phi^{n+\frac{i}{2}}=\phi_{i}\left(t_{n+1}\right)$, for $i=1,2$. It can be shown that this method is first-order accurate in time, see e.g., [27].

We apply this approach to split the problem (3.6)-(3.9) into two subproblems: a structure and a fluid sub-problem defining the operators $A_{1}$ and $A_{2}$.

Similarly as in $[43,42]$, we use semi-discretization in time to define a sequence of approximate solutions to Problem 3.1. This approach defines a time step, which will be denoted by $\Delta t$, and a number of time sub-intervals $N \in \mathbb{N}$, so that

$$
(0, T)=\cup_{n=0}^{N-1}\left(t^{n}, t^{n+1}\right), \quad t^{n}=n \Delta t, n=0, \ldots, N-1 .
$$

For every subdivision containing $N \in \mathbb{N}$ sub-intervals, we recursively define the vector of unknown approximate solutions

$$
\mathbf{X}_{N}^{n+\frac{i}{2}}=\left(\mathbf{u}_{N}^{n+\frac{i}{2}}, v_{N}^{n+\frac{i}{2}}, \eta_{N}^{n+\frac{i}{2}}\right)^{T}, n=0,1, \ldots, N-1, \quad i=1,2,
$$

where $i=1,2$ denotes the solution of sub-problem A1 or A2, respectively. The initial condition will be denoted by

$$
\mathbf{X}^{0}=\left(\mathbf{u}_{0}, v_{0}, \eta_{0}\right)^{T} .
$$

The semi-discretization and the splitting of the problem will be performed in such a way that the discrete version of the energy inequality (2.21) is preserved at every time step. This is a crucial ingredient for the existence proof.

We define semi-discrete versions of the kinetic and elastic energy, originally defined in (2.22), and of dissipation, originally defined in (2.23), by 
the following:

$$
\begin{aligned}
E_{N}^{n+\frac{i}{2}}= & \frac{1}{2}\left(\rho_{f} \int_{\Omega}\left(R+\eta^{n-1+i}\right)^{2}\left|\mathbf{u}_{N}^{n+\frac{i}{2}}\right|^{2}+\rho_{K} h\left\|v_{N}^{n+\frac{i}{2}}\right\|_{L^{2}(\omega)}^{2}\right. \\
& \left.+\frac{h}{2}\left\|\eta_{N}^{n+\frac{i}{2}}\right\|_{\gamma}^{2}+\frac{h^{3}}{24}\left\|\eta_{N}^{n+\frac{i}{2}}\right\|_{\sigma}^{2}\right) \\
D_{N}^{n+1}= & \Delta t \mu_{F} \int_{\Omega}\left(R+\eta^{n}\right)^{2}\left|D^{\eta^{n}}\left(\mathbf{u}_{N}^{n+1}\right)\right|^{2}, n=0, \ldots, N-1, i=0,1
\end{aligned}
$$

Throughout the rest of this section, we fix the time step $\Delta t$, i.e., we keep $N \in \mathbb{N}$ fixed, and study the semi-discretized sub-problems defined by the Lie splitting. To simplify notation, we will omit the subscript $N$ and write $\left(\mathbf{u}^{n+\frac{i}{2}}, v^{n+\frac{i}{2}}, \eta^{n+\frac{i}{2}}\right)$ instead of $\left(\mathbf{u}_{N}^{n+\frac{i}{2}}, v_{N}^{n+\frac{i}{2}}, \eta_{N}^{n+\frac{i}{2}}\right)$.

The splitting that "preserves" the energy estimate and provides a stable, convergent semi-discrete scheme is based on the following operators $A 1$ and $A 2$ :

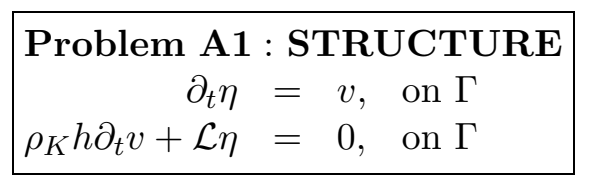

$$
\begin{aligned}
\text { Problem A2 : FLUID } & \text { in } \Omega \\
\partial_{t} \mathbf{u}+\left((\hat{\mathbf{u}}-\mathbf{w}) \cdot \nabla^{\eta}\right) \mathbf{u} & =\nabla^{\eta} \cdot \boldsymbol{\sigma}^{\eta}, \\
\nabla^{\eta} \cdot \mathbf{u} & =0 \\
\text { with : } & \\
\left.\mathbf{u}\right|_{\Gamma} & =v \mathbf{e}_{r}, \\
\rho_{K} h \partial_{t} v+\left.J \boldsymbol{\sigma}^{\eta} \mathbf{n}\right|_{\Gamma} \cdot \mathbf{e}_{r} & =0
\end{aligned}
$$

Here $\hat{\mathbf{u}}$ is the value of $\mathbf{u}$ from the previous time step, and $\mathbf{w}$, which is the domain velocity (the time derivative of the ALE mapping), is obtained from the just calculated Problem A1. Furthermore, $\nabla^{\eta}$ is the transformed gradient, which is based on the value of $\eta$ from the previous time-step. The initial data for $\mathbf{u}$ is given from the previous time step, while the initial data for the trace of the fluid velocity $v$ is given by the just calculated velocity of the thin structure $\partial_{t} \eta$ in Problem $A 1$.

Notice the Robin-type boundary condition on $\Gamma$ for the fluid sub-problem, involving structure inertia $\rho_{K} h \partial_{t} v$. The inclusion of the structure inertia in the boundary condition for the fluid sub-problem is the main reason why the resulting operator slitting scheme is stable. This contrasts the classical Dirichlet-Neumann partitioned schemes in which the boundary condition for the fluid sub-problem is the classical Dirichlet condition, enforcing the fluid velocity on $\Gamma$ to be equal to the velocity of the fluid-structure interface, obtained from the previous time step. It was shown in [8] that this approach gives rise to an unconditionally unstable partitioned, loosely coupled scheme 
when the fluid and structure densities are comparable. In this manuscript we show that the splitting, proposed above, gives rise to a stable, convergent scheme whose approximate, semi-discretized solutions converge to a weak solution to the fully coupled, nonlinear FSI problem in 3D.

Details of the semi-discretized problems in weak form are given next. As we shall see below, each of the semi-discretized problems, defined by the splitting mentioned above, defines a linear problem of eliptic type, for which the existence of a unique solution is guaranteed by the Lax-Milgram Lemma. The main issue, however, is to show that the solutions of the semi-discretized problems satisfy the energy estimates which, when combined together, give rise to a uniform energy estimate of the coupled, semi-discretized problem. It is this uniform boundedness that will give rise to convergent sub-sequences whose limits (weak, or weak*) will define weak solutions to the coupled FSI problem.

4.1.1. Problem A1: the structure elastodynamics problem We write a semi-discrete version of Problem A1 (Structure Elastodynamics). In this step $\mathbf{u}$ does not change, and so

$$
\mathbf{u}^{n+\frac{1}{2}}=\mathbf{u}^{n}
$$

We define $\left(v^{n+\frac{1}{2}}, \eta^{n+\frac{1}{2}}\right) \in H_{0}^{2}(\omega) \times H_{0}^{2}(\omega)$ as a solution of the following problem, written in weak form: for all $\psi \in H_{0}^{2}(\omega)$ solve

$$
\begin{gathered}
\int_{\omega} \frac{\eta^{n+\frac{1}{2}}-\eta^{n}}{\Delta t} \phi=\int_{\omega} v^{n+\frac{1}{2}} \phi, \quad \phi \in L^{2}(\omega), \\
\rho_{K} h \int_{\omega} \frac{v^{n+\frac{1}{2}}-v^{n}}{\Delta t} \psi+\frac{h}{2} \int_{\omega} \mathcal{A} \gamma\left(\eta^{n+\frac{1}{2}}\right): \gamma(\psi)+\frac{h^{3}}{24} \int_{\omega} \mathcal{A} \boldsymbol{\rho}\left(\eta^{n+\frac{1}{2}}\right): \boldsymbol{\rho}(\psi)=0 .
\end{gathered}
$$

The first equation is a weak form of the semi-discretized kinematic coupling condition, while the second equation corresponds to a weak form of the semi-discretized elastodynamics equation.

We will be assuming that the Lamé constants are such that operator $\mathcal{A}$ is coercive, e.g. $\lambda, \mu>0$.

Proposition 4.1. For each fixed $\Delta t>0$, problem (4.4) with $\lambda, \mu>0$ has a unique solution $\left(v^{n+\frac{1}{2}}, \eta^{n+\frac{1}{2}}\right) \in H_{0}^{2}(\omega) \times H_{0}^{2}(\omega)$.

Notice that the semi-discretized problem is a linear elliptic problem, and so we can use the Lax-Milgram Lemma to show the existence of a unique solution. See [43] for more details. 
Proposition 4.2. For each fixed $\Delta t>0$, solution of problem (4.4) satisfies the following discrete energy equality:

$$
E_{N}^{n+\frac{1}{2}}+\frac{1}{2}\left(\rho_{K} h\left\|v^{n+\frac{1}{2}}-v^{n}\right\|^{2}+\frac{h}{2}\left\|\eta^{n+\frac{1}{2}}-\eta^{n}\right\|_{\sigma}^{2}+\frac{h^{3}}{24}\left\|\eta^{n+\frac{1}{2}}-\eta^{n}\right\|_{\sigma}^{2}\right)=E_{N}^{n},
$$

where the kinetic energy $E_{N}^{n}$ is defined in (4.2).

The proof is similar to the corresponding proof presented in [43].

4.1.2. Problem A2: the fluid problem In this step $\eta$ does not change, and so

$$
\eta^{n+1}=\eta^{n+\frac{1}{2}}
$$

Define $\left(\mathbf{u}^{n+1}, v^{n+1}\right) \in \mathcal{V}_{F}^{\eta^{n}} \times L^{2}(\omega)$ by requiring that for all $(\mathbf{q}, \psi) \in \mathcal{V}_{F}^{\eta^{n}} \times$ $L^{2}(\omega)$ such that $\mathbf{q}_{\mid \Gamma}=\psi \mathbf{e}_{r}$, the following weak formulation holds:

$$
\begin{gathered}
\rho_{f} \int_{\Omega}\left(R+\eta^{n}\right)^{2}\left(\frac{\mathbf{u}^{n+1}-\mathbf{u}^{n+\frac{1}{2}}}{\Delta t} \cdot \mathbf{q}+\frac{1}{2}\left[\left(\mathbf{u}^{n}-\mathbf{w}^{n+\frac{1}{2}}\right) \cdot \nabla^{\eta^{n}}\right] \mathbf{u}^{n+1} \cdot \mathbf{q}\right. \\
\left.-\frac{1}{2}\left[\left(\mathbf{u}^{n}-\mathbf{w}^{n+\frac{1}{2}}\right) \cdot \nabla^{\eta^{n}}\right] \mathbf{q} \cdot \mathbf{u}^{n+1}\right)+\rho_{f} \int_{\Omega}\left(R+\frac{\eta^{n}+\eta^{n+1}}{2}\right) v^{n+\frac{1}{2}} \mathbf{u}^{n+1} \cdot \mathbf{q} \\
+2 \mu \int_{\Omega}\left(R+\eta^{n}\right)^{2} \mathbf{D}^{\eta^{n}}(\mathbf{u}): \mathbf{D}^{\eta^{n}}(\mathbf{q})+R \rho_{K} h \int_{\omega} \frac{v^{n+1}-v^{n+\frac{1}{2}}}{\Delta t} \psi \\
=R\left(P_{i n}^{n} \int_{0}^{1}\left(q_{z}\right)_{\mid z=0}-P_{\text {out }}^{n} \int_{0}^{1}\left(q_{z}\right)_{\mid z=L}\right), \\
\text { with } \nabla^{\eta^{n}} \cdot \mathbf{u}^{n+1}=0, \quad \mathbf{u}_{\mid \Gamma}^{n+1}=v^{n+1} \mathbf{e}_{r},
\end{gathered}
$$

where $P_{\text {in } / \text { out }}^{n}=\frac{1}{\Delta t} \int_{n \Delta t}^{(n+1) \Delta t} P_{\text {in } / \text { out }}(t) d t$ and $\mathbf{w}^{n+\frac{1}{2}}=v^{n+\frac{1}{2}}(0, \tilde{x}, \tilde{y})^{t}$.

The main differences between weak formulation (4.6) and the weak formulation of the fluid subproblem in [43] are the spatial dimension, and the Jacobian of the ALE mapping. In particular, this refers to the fourth term in (4.6) which comes from our particular discretization of $-\rho_{f} \int_{0}^{T} \int_{\Omega}(R+$ $\eta)^{2}\left(\nabla^{\eta} \cdot \mathbf{w}^{\eta}\right) \mathbf{u} \cdot \mathbf{q}$. More precisely, $\nabla^{\eta} \cdot \mathbf{w}^{\eta}$, was discretized as

$$
\nabla^{\eta} \cdot \mathbf{w}^{\eta}=\left(R+\frac{\eta^{n}+\eta^{n+1}}{2}\right) v^{n+\frac{1}{2}}
$$

taking into account the kinematic coupling condition $\partial_{t} \eta=v$. This term measures the change of volume of the fluid domain due to the motion of the boundary. Notice that in this term we have a contribution of the Jacobian 
(unlike in the $2 D$ case), which is discretized in a very particular way $(R+$ $\left.\frac{\eta^{n}+\eta^{n+1}}{2}\right)$. The importance of such a discretization will be revealed in the proof of the discrete energy estimates. It is related to the discretization of the Jacobian of the ALE mapping so that the resulting scheme satisfies the geometric conservation law property. See [24] for more details on geometric conservation laws and ALE mappings.

Proposition 4.3. Let $\Delta t>0$, and assume that $\eta^{n}$ are such that $R+\eta^{n} \geq$ $R_{\min }>0, n=0, \ldots, N$. Then, the fluid sub-problem defined by (4.6) has a unique weak solution $\left(\mathbf{u}^{n+1}, v^{n+1}\right) \in \mathcal{V}_{F}^{\eta^{n}} \times L^{2}(\omega)$.

Proof. Notice again that this is a linear, elliptic problem, and the proof of the existence of a unique solution is obtained by using the Lax-Milgram Lemma. The continuity of the operator is proved by using the Sobolev embedding of $H^{1}(\Omega)$ into $L^{4}(\Omega)$, which is valid both in $2 D$ and in $3 D$. Details of the proof can be found in Proposition 3 in [43].

Proposition 4.4. For each fixed $\Delta t>0$, solution of problem (4.6) satisfies the following discrete energy inequality:

$$
\begin{gathered}
E_{N}^{n+1}+\frac{\rho_{f}}{2} \int_{\Omega}\left(R+\eta^{n}\right)^{2}\left|\mathbf{u}^{n+1}-\mathbf{u}^{n}\right|^{2}+\frac{\rho_{K} h}{2}\left\|v^{n+1}-v^{n+\frac{1}{2}}\right\|_{L^{2}(\omega)}^{2} \\
+D_{N}^{n+1} \leq E_{N}^{n+\frac{1}{2}}+C \Delta t\left(\left(P_{\text {in }}^{n}\right)^{2}+\left(P_{\text {out }}^{n}\right)^{2}\right),
\end{gathered}
$$

where the kinetic energy $E_{N}^{n}$ and dissipation $D_{N}^{n}$ are defined in (4.2) and (4.3), and the constant $C$ depends only on the parameters in the problem, and not on $\Delta t$ (or $N)$.

Proof. We begin by focusing on the weak formulation (4.6) in which we replace the test functions $\mathbf{q}$ by $\mathbf{u}^{n+1}$ and $\psi$ by $v^{n+1}$. We multiply the resulting equation by $\Delta t$, and notice that the first term on the right hand-side is given by

$$
\frac{\rho_{f}}{2} \int_{\Omega}\left(R+\eta^{n}\right)^{2}\left|\mathbf{u}^{n+1}\right|^{2} .
$$

This is the term that contributes to the discrete kinetic energy at the time step $n+1$, but it does not have the correct form, since the discrete kinetic energy at $n+1$ is given in terms of the structure location at $n+1$, and not at $n$, namely, the discrete kinetic energy at $n+1$ involves

$$
\frac{\rho_{f}}{2} \int_{\Omega}\left(R+\eta^{n+1}\right)^{2}\left|\mathbf{u}^{n+1}\right|^{2} .
$$


To get around this difficulty it is crucial that the advection term is present in the fluid sub-problem. The advection term is responsible for the presence of the integral

$$
\rho_{f} \int_{\Omega}\left(R+\frac{\eta^{n}+\eta^{n+1}}{2}\right) \Delta t v^{n+\frac{1}{2}}\left|\mathbf{u}^{n+1}\right|^{2}
$$

which can be re-written by noticing that $\Delta t v^{n+\frac{1}{2}}:=\left(\eta^{n+1 / 2}-\eta^{n}\right)$ which is equal to $\left(\eta^{n+1}-\eta^{n}\right)$ since, in this sub-problem $\eta^{n+1}=\eta^{n+1 / 2}$. This implies

$$
\begin{aligned}
& \frac{\rho_{f}}{2}\left(\int_{\Omega}\left(R+\eta^{n}\right)^{2}\left|\mathbf{u}^{n+1}\right|^{2}+2\left(R+\frac{\eta^{n}+\eta^{n+1}}{2}\right) \Delta t v^{n+\frac{1}{2}}\left|\mathbf{u}^{n+1}\right|^{2}\right) \\
& \quad=\frac{\rho_{f}}{2} \int_{\Omega}\left(R+\eta^{n+1}\right)^{2}\left|\mathbf{u}^{n+1}\right|^{2} .
\end{aligned}
$$

Thus, these two terms combined provide the discrete kinetic energy at the time step $n+1$. It is interesting to notice how the nonlinearity of the coupling at the deformed boundary requires the presence of nonlinear advection in order for the discrete kinetic energy of the fluid sub-problem to be decreasing in time, and to thus satisfy the desired energy estimate. The rest of the proof is the same as that presented in [43], and is based on the use algebraic identity $(a-b) \cdot a=\frac{1}{2}\left(|a|^{2}+|a-b|^{2}-|b|^{2}\right)$.

We pause for a second, and summarize what we have accomplished so far. For a given $\Delta t>0$ we divided the time interval $(0, T)$ into $N=T / \Delta t$ sub-intervals $\left(t^{n}, t^{n+1}\right), n=0, \ldots, N-1$. On each sub-interval $\left(t^{n}, t^{n+1}\right)$ we "solved" the coupled FSI problem by applying the Lie splitting scheme. First we solved for the structure position (Problem A1) and then for the fluid flow (Problem A2). We have just shown that each sub-problem has a unique solution, provided that $R+\eta^{n} \geq R_{\min }>0, n=0, \ldots, N$, and that its solution satisfies an energy estimate. When combined, the two energy estimates provide a discrete version of the energy estimate (2.21). Thus, for each $\Delta t$ we have a time-marching, splitting scheme which defines an approximate solution on $(0, T)$ of our main FSI problem defined in Problem 3.1, and is such that for each $\Delta t$ the approximate FSI solution satisfies a discrete version of the energy estimate for the continuous problem.

What we would like to ultimately show is that, as $\Delta t \rightarrow 0$, the sequence of solutions parameterized by $N$ (or $\Delta t$ ), converges to a weak solution of Problem 3.1. Furthermore, we also need to show that $R+\eta^{n} \geq R_{\min }>0$ is satisfied for each $n=0, \ldots, N-1$. In order to obtain this result, it is crucial to show that the discrete energy of the approximate FSI solutions defined for each $\Delta t$, is uniformly bounded, independently of $\Delta t$ (or $N$ ). This result is obtained by the following Lemma. 
Lemma 4.1. (The uniform energy estimates) Let $\Delta t>0$ and $N=$ $T / \Delta t>0$. Furthermore, let $E_{N}^{n+\frac{1}{2}}, E_{N}^{n+1}$, and $D_{N}^{j}$ be the kinetic energy and dissipation given by (4.2) and (4.3), respectively.

There exists a constant $C>0$ independent of $\Delta t$ (and $N$ ), which depends only on the parameters in the problem, on the kinetic energy of the initial data $E_{0}$, and on the energy norm of the inlet and outlet data $\left\|P_{\text {in/out }}\right\|_{L^{2}(0, T)}^{2}$, such that the following estimates hold:

(1) $E_{N}^{n+\frac{1}{2}} \leq C, E_{N}^{n+1} \leq C$, for all $n=0, \ldots, N-1$,

(2) $\sum_{j=1}^{N} D_{N}^{j} \leq C$,

(3) $\sum_{n=0}^{N-1}\left(\int_{\Omega}\left(R+\eta^{n}\right)^{2}\left|\mathbf{u}^{n+1}-\mathbf{u}^{n}\right|^{2}+\left\|v^{n+1}-v^{n+\frac{1}{2}}\right\|_{L^{2}(\omega)}^{2}\right.$ $\left.+\left\|v^{n+\frac{1}{2}}-v^{n}\right\|_{L^{2}(\omega)}^{2}\right) \leq C$,

(4) $\sum_{n=0}^{N-1}\left(\left\|\eta^{n+1}-\eta^{n}\right\|_{\gamma}+\left\|\eta^{n+1}-\eta^{n}\right\|_{\sigma}\right) \leq C$.

In fact, $C=E_{0}+\tilde{C}\left(\left\|P_{\text {in }}\right\|_{L^{2}(0, T)}^{2}+\left\|P_{\text {out }}\right\|_{L^{2}(0, T)}^{2}\right)$, where $\tilde{C}$ is the constant from (4.7), which depends only on the parameters in the problem.

Proof. The proof is analogous to the proof of Lemma 1 in [43]. It is a consequence of the energy estimates (4.5) and (4.7).

\section{Convergence of approximate solutions}

Unlike in the radially symmetric $2 \mathrm{D}$ case studied in [43], the convergence of approximate solutions to a weak solution of the full 3D problem without radial symmetry requires the use of more sophisticated techniques than those used in [43]. The main reasons for that are the fact that in 3D the fluidstructure interface $\Gamma$ is not necessarily Lipschitz, and that different Sobolev embedding theorems imply different regularity of solutions than in the $2 \mathrm{D}$ case. While we omit details that are similar to those in the 2D case, in this section we pay particular attention to the differences with the $2 \mathrm{D}$ radially symmetric case and present the details whenever necessary.

We define approximate solutions of Problem 3.1 on $(0, T)$ to be the functions which are piece-wise constant on each sub-interval $((n-1) \Delta t, n \Delta t], n=$ $1 \ldots N$ of $(0, T)$, such that for $t \in((n-1) \Delta t, n \Delta t], n=1 \ldots N$,

$$
\mathbf{u}_{N}(t, .)=\mathbf{u}_{N}^{n}, \eta_{N}(t, .)=\eta_{N}^{n}, v_{N}(t, .)=v_{N}^{n}, v_{N}^{*}(t, .)=v_{N}^{n-\frac{1}{2}} .
$$

See Figure 3 left. Notice that functions $v_{N}^{*}=v_{N}^{n-1 / 2}$ are determined by Step A1 (the elastodynamics sub-problem), while functions $v_{N}=v_{N}^{n}$ are 

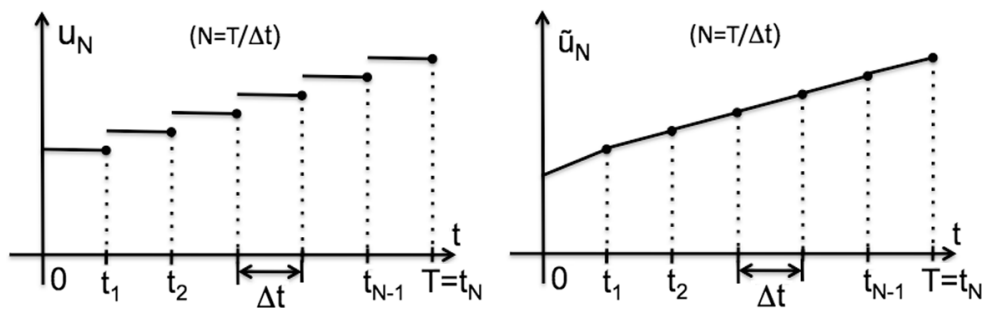

Figure 3: A sketch of two different approximations of $u_{N}$ used in the existence proof: a piece-wise constant approximation $u_{N}$ (left), and a piece-wise linear approximation $\tilde{u}_{N}$ (right).

determined by Step A2 (the fluid sub-problem). As a consequence, functions $v_{N}$ are equal to the normal trace of the fluid velocity on $\Gamma$, i.e., $\mathbf{u}_{\mathbf{N}}=$ $v_{N} \mathbf{e}_{\mathbf{r}}$. This is not necessarily the case for the functions $v_{N}^{*}$. However, we will show later that the difference between the two sequences converges to zero in $L^{2}$.

Using Lemma 4.1 we now show that these sequences are uniformly bounded in the appropriate solution spaces. We begin by showing that $\left(\eta_{N}\right)_{N \in \mathbb{N}}$ is uniformly bounded in $L^{\infty}\left(0, T ; H_{0}^{2}(\omega)\right)$, and that there exists a $T>0$ for which $R+\eta_{N}^{n}>0$ holds independently of $N$ and $n$. This implies, among other things, that our approximate solutions are, indeed, well-defined on a non-zero time interval $(0, T)$.

Proposition 5.1. Sequence $\left(\eta_{N}\right)_{N \in \mathbb{N}}$ is uniformly bounded in

$$
L^{\infty}\left(0, T ; H_{0}^{2}(\omega)\right)
$$

Moreover, for T small enough, we have

$$
0<R_{\min } \leq R+\eta_{N}(t, z, \theta) \leq R_{\max }, \forall N \in \mathbb{N},(z, \theta) \in \omega, t \in(0, T)
$$

Proof. From Lemma 4.1 we have that $E_{N}^{n} \leq C$, where $C$ is independent of $N$. This implies

$$
\left\|\eta_{N}(t)\right\|_{\sigma}^{2}+\left\|\eta_{N}(t)\right\|_{\gamma}^{2} \leq C, \forall t \in[0, T]
$$

Therefore, from the equivalence of norms $\|\cdot\|_{H_{0}^{2}}$ and $\|\cdot\|_{\sigma}$ we have

$$
\left\|\eta_{N}\right\|_{L^{\infty}\left(0, T ; H_{0}^{2}(\omega)\right)} \leq C
$$


To show that the radius $R+\eta_{N}$ is uniformly bounded away from zero for $T$ small enough, we first notice that the above inequality implies

$$
\left\|\eta_{N}^{n}-\eta_{0}\right\|_{H_{0}^{2}(\omega)} \leq 2 C, n=1, \ldots, N, N \in \mathbb{N} .
$$

Furthermore, we calculate

$$
\left\|\eta_{N}^{n}-\eta_{0}\right\|_{L^{2}(\omega)} \leq \sum_{i=0}^{n-1}\left\|\eta_{N}^{i+1}-\eta_{N}^{i}\right\|_{L^{2}(\omega)}=\Delta t \sum_{i=0}^{n-1}\left\|v_{N}^{i+\frac{1}{2}}\right\|_{L^{2}(\omega)},
$$

where we recall that $\eta_{N}^{0}=\eta_{0}$. From Lemma 4.1 we have that $E_{N}^{n+\frac{1}{2}} \leq C$, where $C$ is independent of $N$. This combined with the above inequality implies

$$
\left\|\eta_{N}^{n}-\eta_{0}\right\|_{L^{2}(\omega)} \leq C n \Delta t \leq C T, n=1, \ldots, N, N \in \mathbb{N} .
$$

Now, we have uniform bounds for $\left\|\eta_{N}^{n}-\eta_{0}\right\|_{L^{2}(\omega)}$ and $\left\|\eta_{N}^{n}-\eta_{0}\right\|_{H_{0}^{2}(\omega)}$. Therefore, we can use the interpolation inequality for Sobolev spaces (see for example [1], Thm. 4.17, p. 79) to get

$$
\left\|\eta_{N}^{n}-\eta_{0}\right\|_{H^{3 / 2}(\omega)} \leq C T^{1 / 4}, n=1, \ldots, N, N \in \mathbb{N} .
$$

From Lemma 4.1 we see that $C$ depends on $T$ through the norms of the inlet and outlet data in such a way that $C$ is an increasing function of $T$. Therefore by choosing $T$ small, we can make $\left\|\eta_{N}^{n}-\eta_{0}\right\|_{H^{3 / 2}(\omega)}$ arbitrary small for $n=1, \ldots, N, N \in \mathbb{N}$. Because of the Sobolev embedding of $H^{3 / 2}(\omega)$ into $C(\bar{\omega})$ we can also make $\left\|\eta_{N}^{n}-\eta_{0}\right\|_{C(\omega)}$ arbitrarily small. Since the initial data $\eta_{0}$ is such that $R+\eta_{0}(z, \theta)>0$ (due to the conditions listed in (2.15)), we see that for a $T>0$ small enough, there exist $R_{\min }, R_{\max }>0$, such that

$$
0<R_{\min } \leq R+\eta_{N}(t, z, \theta) \leq R_{\max }, \forall N \in \mathbb{N},(z, \theta) \in \omega, t \in(0, T) .
$$

We will show in the end that our existence result holds not only locally in time, i.e., for small $T>0$, but rather, it can be extended all the way until either $T=\infty$, or until the lateral walls of the channel touch each other.

From this Proposition we see that the $L^{2}$-norm $\|f\|_{L^{2}(\Omega)}^{2}=\int f^{2}$, and the weighted $L^{2}$-norm $\|f\|_{L^{2}(\Omega)}^{2}=\int\left(R+\eta_{N}\right)^{2} f^{2}$ are equivalent. More precisely, for every $f \in L^{2}(\Omega)$, there exist constants $C_{1}, C_{2}>0$, which depend only on $R_{\min }, R_{\max }$, and not on $f$ or $N$, such that

$$
C_{1} \int_{\Omega}\left(R+\eta_{N}\right)^{2} f^{2} \leq\|f\|_{L^{2}(\Omega)}^{2} \leq C_{2} \int_{\Omega}\left(R+\eta_{N}\right)^{2} f^{2} .
$$


We will be using this property in the next section to prove strong convergence of approximate functions.

Next we show that the sequences of approximate solutions for the velocity and its trace on the lateral boundary, are uniformly bounded. To do that, we introduce the following notation which will be useful in the remainder of this manuscript to prove compactness: denote by $\tau_{h}$ the translation in time by $h$ of a function $f$

$$
\tau_{h} f(t, .)=f(t-h, .), h \in \mathbb{R}
$$

Proposition 5.2. The following statements hold:

(1) $\left(v_{N}\right)_{n \in \mathbb{N}}$ is uniformly bounded in $L^{\infty}\left(0, T ; L^{2}(\omega)\right)$.

(2) $\left(v_{N}^{*}\right)_{n \in \mathbb{N}}$ is uniformly bounded in $L^{\infty}\left(0, T ; L^{2}(\omega)\right)$.

(3) $\left(\mathbf{u}_{N}\right)_{n \in \mathbb{N}}$ is uniformly bounded in $L^{\infty}\left(0, T ; L^{2}(\Omega)\right)$.

(4) $\left(\mathbf{D}^{\tau_{\Delta t} \eta_{N}}\left(\mathbf{u}_{N}\right)\right)_{n \in \mathbb{N}}$ is uniformly bounded in $L^{2}((0, T) \times \Omega)$.

Proof. The results follow directly from Statements 1 and 2 of Lemma 4.1, and from the definition of $\left(v_{N}\right)_{n \in \mathbb{N}},\left(v_{N}^{*}\right)_{N \in \mathbb{N}}$ and $\left(\mathbf{u}_{N}\right)_{N \in \mathbb{N}}$ as step-functions in $t$ so that

$$
\int_{0}^{T}\left\|v_{N}\right\|_{L^{2}(0, L)}^{2} d t=\sum_{n=0}^{N-1}\left\|v_{N}^{n}\right\|_{L^{2}(0, L)}^{2} \Delta t .
$$

Unfortunately, having the boundedness of the symmetrized gradient is not sufficient to show that the approximate solutions coverage to a weak solution of the coupled FSI problem. We need be able to control the behavior of the gradient itself. For this purpose, we prove the following proposition.

Proposition 5.3. The gradient $\left(\nabla^{\tau_{\Delta t} \eta_{N}}\left(\mathbf{u}_{N}\right)\right)_{n \in \mathbb{N}}$ is uniformly bounded in $L^{2}((0, T) \times \Omega)$.

Proof. We use Korn's inequality and the boundedness of the symmetrized gradient to prove this statement. However, Proposition 5.2 shows the boundedness of the transformed symmetrized gradient, and not of the symmetrized gradient itself. To deal with this difficulty, we temporarily map the problem back onto the physical domain $\Omega_{\eta_{N}^{n-1}}$, where the transformed symmetrized gradient becomes the standard symmetrized gradient, and apply Korn's inequality in the usual way. However, since the Korn's constant in general depends on the domain, i.e., on the ALE mapping, we will need a result which gives a universal Korn's constant, independent of the family of domains under consideration. Indeed, we use an approach similar to the one presented in [42] (proof of Proposition 6.5, pp. 683-685) to find a universal 
Korn's constant, independent of the ALE mappings, that can be obtained under certain assumptions on the family of domains, which hold true in our case. The calculation presented in [42] was done in the spirit similar to the work of Chambolle et al. [9], Lemma 6, pp. 377, which was also done in 3D. Similar results, under slightly different assumptions can also be found in $[51,43]$. By using this result we can obtain a universal Korn'a constant, which implies uniform boundedness of the sequence of gradients of $\mathbf{u}_{N}$ on the physical domains $\Omega_{\eta_{v}^{n-1}}$, which, after mapping everything back to the fixed, reference domain $\Omega$ via the ALE mappings, implies uniform boundedness of $\left(\nabla^{\tau_{\Delta t} \eta_{N}}\left(\mathbf{u}_{N}\right)\right)_{n \in \mathbb{N}}$.

From the uniform boundedness of approximate sequences we can now conclude that for each approximate solution sequence there exists a subsequence which, with a slight abuse of notation, we denote the same way as the original sequence, and which converges weakly, or weakly*, depending on the function space. More precisely, we have the following result.

Lemma 5.1. (Weak and weak* convergence results) There exist subsequences $\left(\eta_{N}\right)_{N \in \mathbb{N}},\left(v_{N}\right)_{N \in \mathbb{N}},\left(v_{N}^{*}\right)_{N \in \mathbb{N}}$, and $\left(\mathbf{u}_{N}\right)_{N \in \mathbb{N}}$, and the functions $\eta \in$ $L^{\infty}\left(0, T ; H_{0}^{2}(\omega)\right), v \in L^{\infty}\left(0, T ; L^{2}(\omega)\right) \cap L^{2}\left(0, T ; H_{0}^{2}(\omega)\right), v^{*} \in L^{\infty}(0, T ;$ $\left.L^{2}(\omega)\right)$, and $\mathbf{u} \in L^{\infty}\left(0, T ; L^{2}(\Omega)\right)$, such that

$$
\begin{aligned}
\eta_{N} & \rightarrow \eta \text { weakly* in } L^{\infty}\left(0, T ; H_{0}^{2}(\omega)\right), \\
v_{N} & \rightarrow v \text { weakly in } L^{2}\left(0, T ; L^{2}(\omega)\right), \\
v_{N} & \rightarrow v \text { weakly* in } L^{\infty}\left(0, T ; L^{2}(\omega)\right), \\
v_{N}^{*} & \rightarrow v^{*} \text { weakly* in } L^{\infty}\left(0, T ; L^{2}(\omega)\right), \\
\mathbf{u}_{N} & \rightarrow \mathbf{u} \text { weakly* in } L^{\infty}\left(0, T ; L^{2}(\Omega)\right), \\
\nabla^{\tau_{\Delta t} \eta_{N}} \mathbf{u}_{N} & \rightarrow \text { G weakly in } L^{2}((0, T) \times \Omega) .
\end{aligned}
$$

Furthermore,

$$
v=v^{*} \text {. }
$$

Proof. The only thing left to show is that $v=v^{*}$. To show this, we multiply statement (3) in Lemma 4.1 by $\Delta t$, and notice again that $\left\|v_{N}\right\|_{L^{2}((0, T) \times \omega)}^{2}=$ $\Delta t \sum_{n=1}^{N}\left\|v_{N}^{n}\right\|_{L^{2}(\omega)}^{2}$. This implies $\left\|v_{N}-v_{N}^{*}\right\|_{L^{2}((0, T) \times \omega)}^{2}=\Delta t \sum_{n=1}^{N} \mid v_{N}^{n}-$ $\left.v_{N}^{n-\frac{1}{2}}\right|^{2} \leq C \Delta t$, and we have that in the limit, as $\Delta t \rightarrow 0, v=v^{*}$.

Naturally, our goal is to prove that $\mathbf{G}=\nabla^{\eta} \mathbf{u}$, where $\eta$ is the limiting displacement determining the fluid-structure interface location. However, to achieve this goal we will need some stronger convergence properties of approximate solutions. Therefore, we postpone the proof until Proposition 6.1. 


\subsection{Strong convergence of approximate sequences}

To show that the limits obtained in the previous Lemma satisfy the weak form of Problem 3.1, we will need to show that our sequences converge strongly in the appropriate function spaces.

Theorem 5.1. Sequences $\left(v_{N}\right)_{N \in \mathbb{N}},\left(\mathbf{u}_{N}\right)_{N \in \mathbb{N}}$ are relatively compact in $L^{2}\left(0, T ; L^{2}(\omega)\right)$ and $L^{2}\left(0, T ; L^{2}(\Omega)\right)$ respectively.

Proof. The proof is based on Simon's theorem which characterizes compact sets in $L^{p}(0, T ; X)$, where $X$ is a Banach space [47]. See also [43] where details of the use of Simon's theorem in the proof of compactness in the 2D radially symmetric case are presented. Simon's theorem says that for a set $F$, $F \hookrightarrow L^{p}(0, T ; X)$, with $1 \leq p<\infty$ to be relatively compact in $L^{p}(0, T ; X)$, it is necessary and sufficient that the following two properties are satisfied:

(i) $\left\|\tau_{h} f-f\right\|_{L^{p}(h, T ; X)} \rightarrow 0$ as $h$ goes to zero, uniformly in $f \in F$ (integral "equicontinuity" in time), and

(ii) $\left\{\int_{t_{1}}^{t_{2}} f(t) d t: f \in F\right\}$ is relatively compact in $X, 0<t_{1}<t_{2}<T$ (spatial compactness).

To show the "integral equicontinuity" estimate in time, we need to show:

$$
\left\|\tau_{h} v_{N}-v_{N}\right\|_{L^{2}(0, T \times \omega)} \rightarrow 0, h \rightarrow 0, \text { uniformly } \in N
$$

Thus, we want to show that for each $\varepsilon>0$, there exists a $\delta>0$ such that

$$
\left\|\tau_{h} v_{N}-v_{N}\right\|_{L^{2}\left(K ; L^{2}(\omega)\right)}^{2}<\varepsilon, \quad \forall|h|<\delta, \text { independently of } N \in \mathbb{N}
$$

where $K$ is an arbitrary compact subset of $\Omega$. Indeed, we will show that for each $\varepsilon>0$, the following choice of $\delta$ :

$$
\delta:=\min \{\operatorname{dist}(K, \partial \Omega) / 2, \varepsilon /(2 C)\}
$$

provides the desired estimate, where $C$ is the constant from Lemma 4.1 (independent of $N$ ). The uniform bounds on the kinetic energy due to the motion of the fluid domain in time, presented in the third equality of Lemma 4.1, are the main reasons why the equicontinuity in time is satisfied by the sequence of approximate functions. Namely, if we multiply the third inequality of Lemma 4.1 by $\Delta t$ we get that the "half-order derivative in time" is uniformly bounded:

$$
\left\|\tau_{\Delta t} \mathbf{u}_{N}-\mathbf{u}_{N}\right\|_{L^{2}((0, T) \times \Omega)}^{2}+\left\|\tau_{\Delta t} v_{N}-v_{N}\right\|_{L^{2}((0, T) \times \omega)}^{2} \leq C \Delta t .
$$


This is "almost" the integral equicontinuity (5.7) except that in this estimate $\varepsilon$ depends on $\Delta t$ (i.e., $N$ ), which is not sufficient to show equicontinuity. We need to show that estimate (5.7) holds for all the functions $\left(v_{N}\right)_{N \in \mathbb{N}}$, $\left(\mathbf{u}_{N}\right)_{N \in \mathbb{N}}$, independently of $N \in \mathbb{N}$. This can be achieved in the same way as in [43], Theorem 2 (see also [42]) by considering two cases $(\Delta t \geq h$ and $\Delta t<h)$ that cover all the possibilities. The use of Lemma 4.1 gives again the desired bounds as in [43], and so we omit the details here.

To complete proving relative compactness using Simon's theorem what is left to show is spatial compactness. The fact that we are working in 3D requires the use of different arguments from those presented in [43]. Namely, since the fluid-structure interface $\Gamma$ is not necessarily Lipschitz we need to use the results about functions defined on a domain which is a sub-graph of a Hölder continuous function, to be able to obtain certain regularity of $\mathbf{u}_{N}$ and make sense of its trace on $\Gamma$, presented in [41]. First, from [41] and statement 2 in Lemma 4.1 we get that sequence $\left(\mathbf{u}_{N}\right)_{N \in \mathbb{N}}$ is uniformly bounded in $L^{2}\left(0, T ; H^{s}(\Omega)\right), s<1$. Then, we notice that

$$
v_{N}=\mathbf{u}_{N \mid \Gamma}, N \in \mathbb{N},
$$

and use the result about the trace on a domain which is not Lipschitz obtained in [41] to conclude that the sequence $\left(v_{N}\right)_{N \in \mathbb{N}}$ is uniformly bounded in $L^{2}\left(0, T ; H^{s / 2}(\omega)\right), s<1$. We complete the proof by noticing that $H^{s}(\Omega)$ and $H^{s / 2}(\omega)$ are compactly embedded in $L^{2}(\Omega)$ and $L^{2}(\omega)$, respectively, $0<$ $s<1$, thereby showing spatial compactness of the approximating sequences $\left(v_{N}\right)_{N \in \mathbb{N}}$ and $\left(\mathbf{u}_{N}\right)_{N \in \mathbb{N}}$ in the spaces $L^{2}\left(0, T ; L^{2}(\omega)\right)$ and $L^{2}\left(0, T ; L^{2}(\Omega)\right)$, respectively.

Combined with temporal equicontinuity, shown above, this proves the relative compactness result of $\left(v_{N}\right)_{N \in \mathbb{N}}$ and $\left(\mathbf{u}_{N}\right)_{N \in \mathbb{N}}$ in $L^{2}\left(0, T ; L^{2}(\omega)\right)$ and $L^{2}\left(0, T ; L^{2}(\Omega)\right)$, respectively.

To show compactness of $\left(\eta_{N}\right)_{N \in \mathbb{N}}$ we introduce a slightly different set of approximate functions of $\mathbf{u}, v$, and $\eta$. Namely, for each fixed $\Delta t$ (or $N \in \mathbb{N}$ ), define $\tilde{\mathbf{u}}_{N}, \tilde{\eta}_{N}$ and $\tilde{v}_{N}$ to be continuous, linear on each sub-interval $[(n-1) \Delta t, n \Delta t]$, and such that

$\tilde{\mathbf{u}}_{N}(n \Delta t,)=.\mathbf{u}_{N}(n \Delta t,),. \tilde{v}_{N}(n \Delta t,)=.v_{N}(n \Delta t,),. \tilde{\eta}_{N}(n \Delta t,)=.\eta_{N}(n \Delta t,)$,

where $n=0, \ldots, N$. See Figure 3 right. Using the same approaches as in [43], Section 6, we can show the following strong convergence results: 
Theorem 5.2. There exist subsequences $\left(\eta_{N}\right)_{N \in \mathbb{N}},\left(v_{N}\right)_{N \in \mathbb{N}}$, such that

$$
\begin{aligned}
v_{N} & \rightarrow v \text { in } L^{2}\left(0, T ; L^{2}(\omega)\right), \\
\tau_{\Delta t} \mathbf{u}_{N} & \rightarrow u \text { in } L^{2}\left(0, T ; L^{2}(\Omega)\right), \\
\tau_{\Delta t} v_{N} & \rightarrow v \text { in } L^{2}\left(0, T ; L^{2}(\omega)\right), \\
\eta_{N} & \rightarrow \eta \text { in } L^{\infty}\left(0, T ; H_{0}^{s}(\omega)\right), s<2 \\
\tau_{\Delta t} \eta_{N} & \rightarrow \eta \text { in } L^{\infty}\left(0, T ; H_{0}^{s}(\omega)\right), s<2 \\
\tilde{\mathbf{u}}_{N} & \rightarrow \mathbf{u} \text { in } L^{2}\left(0, T ; L^{2}(\Omega)\right), \\
\tilde{v}_{N} & \rightarrow v \text { in } L^{2}\left(0, T ; L^{2}(0, L)\right) .
\end{aligned}
$$

From the Sobolev embedding $H^{s}(\omega)$ into $C(\bar{\omega}), s>1$, we get the following strong convergence results for the approximations of the fluid-structure interface $\Gamma$.

\section{Corollary 5.1.}

$$
\begin{aligned}
\eta_{N} & \rightarrow \eta \text { in } L^{\infty}(0, T ; C(\omega)), \\
\tau_{\Delta t} \eta_{N} & \rightarrow \quad \eta \text { in } L^{\infty}(0, T ; C(\omega)) .
\end{aligned}
$$

These strong convergence results will be used to show convergence of our approximate solutions to a weak solution of the fluid-structure interaction Problem 2.1, as $N \rightarrow \infty$.

\section{The limiting problem}

Next we want to show that the limiting functions satisfy the weak form (3.18) of Problem 2.1. In this vein, one of the things that needs to be considered is what happens in the limit as $N \rightarrow \infty$, i.e., as $\Delta t \rightarrow 0$, of problem (4.6). Before we pass to the limit we must observe that, unfortunately, the velocity test functions in (4.6) depend of $N$ ! More precisely, they depend on $\eta_{N}^{n}$ because of the requirement that the transformed divergence-free condition $\nabla \eta_{N}^{n} \cdot \mathbf{q}=0$ must be satisfied. This is a consequence of the fact that we mapped our problem onto a fixed domain $\Omega$. Therefore we will need to take special care in constructing the suitable velocity test functions so that we can pass to the limit in (4.6). This was done in [42], Section 7, and also in $[43,9]$, and so we omit the details of construction here. Therefore, from $[42,43,9]$ we know that there exists a set of test function $\mathcal{X}^{\eta}(0, T)$ which is dense in $\mathcal{Q}^{\eta}(0, T)$, in which the test functions are independent of $N$, and are well approximated by the test functions $\mathbf{q}_{N}$, satisfying the following properties:

- $\mathcal{X}^{\eta}(0, T)$ is dense in $\mathcal{Q}^{\eta}(0, T)$, 
- For every $(\mathbf{q}, \psi) \in \mathcal{X}^{\eta}(0, T)$ there exists a $N_{q} \in \mathbb{N}$ and a sequence $\left(\mathbf{q}_{N}\right)_{N \geq N_{q}}$ such that $\mathbf{q}_{N} \in \mathcal{W}_{F}^{\tau_{\Delta t} \eta}(0, T)$, and

(1) $\mathbf{q}_{N} \rightarrow \mathbf{q}$ uniformly on $[0, T] \times \Omega$;

(2) $\nabla^{\tau_{\Delta t} \eta_{N}}\left(\mathbf{q}_{N}\right) \rightarrow \nabla^{\eta}(\mathbf{q})$ in $L^{2}((0, T) \times \omega)$.

We are now almost ready to pass to the limit in the weak formulation of the coupled FSI problem. The only thing left is to show that the gradients of the approximate velocities converge to the gradient of the limiting velocity, namely, it remains to identify the function $\mathbf{G}$ introduced in Lemma 5.1. We have the following result:

Proposition 6.1. $\mathbf{G}=\nabla^{\eta} \mathbf{u}$, where $\mathbf{G}, \mathbf{u}$ and $\eta$ are the weak and weak* limits given by Lemma 5.1.

Proof. The proof is analogous to the proof of Proposition 7.6. in [42]. Although the proof in [42] is given is a different settings ( $2 D$ vs. $3 D)$ it essentially relies on the following two facts that hold true in both scenarios:

- Uniform convergence of the sequence $\tau_{\Delta t} \eta_{N}$ which is given by Corollary 5.1 ; and

- The proof uses the approximate fluid velocities and the limiting fluid velocity transformed back onto the physical domains:

$$
\mathbf{u}^{N}(t, .)=\mathbf{u}_{N}(t, .) \circ A_{\tau_{\Delta t} \eta_{N}}^{-1}(t), \quad \tilde{\mathbf{u}}(t, .)=\mathbf{u}(t, .) \circ A_{\eta}^{-1}(t),
$$

as well as the fact that $\nabla \mathbf{u}^{N}=\nabla^{\tau_{\Delta t} \eta_{N}} \mathbf{u}_{N}$ and $\nabla \tilde{\mathbf{u}}=\nabla^{\eta} \mathbf{u}$.

Both of these are satisfied in the present case. Since the proof is rather technical we omit the details and refer the reader to [42].

To get to the weak formulation of the coupled problem, take the test functions $\left(\mathbf{q}_{N}(t), \psi(t)\right)$ (where $\mathbf{q}_{N}$ is a sequence of test function corresponding to $\left.(\mathbf{q}, \psi) \in \mathcal{X}^{\eta}\right)$ in equation (4.6) and integrate with respect to $t$ from $n \Delta t$ to $(n+1) \Delta t$. Furthermore, take $\psi(t)$ as the test functions in (4.4), and again integrate over the same time interval. Add the two equations together, and take the sum from $n=0, \ldots, N-1$ to get the time integrals over $(0, T)$ as follows:

$$
\begin{aligned}
\rho_{f} & \int_{0}^{T} \int_{\Omega}\left(R+\tau_{\Delta t} \eta_{N}\right)^{2}\left(\partial_{t} \tilde{\mathbf{u}}_{N} \cdot \mathbf{q}_{N}+\frac{1}{2}\left(\tau_{\Delta t} \mathbf{u}_{N}-\mathbf{w}_{N}\right) \cdot \nabla^{\tau_{\Delta t} \eta_{N}} \mathbf{u}_{N} \cdot \mathbf{q}_{N}\right. \\
& \left.-\frac{1}{2}\left(\tau_{\Delta t} \mathbf{u}_{N}-\mathbf{w}_{N}\right) \cdot \nabla^{\tau_{\Delta t} \eta_{N}} \mathbf{q}_{N} \cdot \mathbf{u}_{N}\right)
\end{aligned}
$$




$$
\begin{aligned}
& +\rho_{f} \int_{0}^{T}\left(R+\frac{1}{2}\left(\tau_{\Delta t} \eta_{N}+\eta_{N}\right)\right) \int_{\Omega} v_{N}^{*} \mathbf{u}_{N} \cdot \mathbf{q}_{N} \\
& +\int_{0}^{T} \int_{\Omega}\left(R+\tau_{\Delta t} \eta_{N}\right)^{2} 2 \mu_{F} \mathbf{D}^{\tau_{\Delta t} \eta_{N}}\left(\mathbf{u}_{\mathbf{N}}\right): \mathbf{D}^{\tau_{\Delta t} \eta_{N}}\left(\mathbf{q}_{N}\right)+R \rho_{K} h \int_{0}^{T} \int_{\omega} \partial_{t} \tilde{v}_{N} \psi \\
& +\frac{R h}{2} \int_{0}^{T} \int_{\omega} \mathcal{A} \gamma(\eta): \gamma(\psi)+\frac{R h^{3}}{24} \int_{0}^{T} \int_{\omega} \mathcal{A} \boldsymbol{\rho}(\eta): \boldsymbol{\rho}(\psi) \\
& =R\left(\int_{0}^{T} P_{\text {in }}^{N} d t \int_{0}^{1} q_{z}(t, 0, r) d r-\int_{0}^{T} P_{\text {out }}^{N} d t \int_{0}^{1} q_{z}(t, L, r) d r\right),
\end{aligned}
$$

with

$$
\begin{gathered}
\nabla^{\tau_{\Delta t} \eta} \cdot \mathbf{u}_{N}=0, \quad v_{N}=\left(\left(u_{r}\right)_{N}\right)_{\mid \Gamma}, \\
\mathbf{u}_{N}(0, .)=\mathbf{u}_{0}, \eta(0, .)_{N}=\eta_{0}, v_{N}(0, .)=v_{0} .
\end{gathered}
$$

Here $\tilde{\mathbf{u}}_{N}$ and $\tilde{v}_{N}$ are the piecewise linear functions defined in (5.10), $\tau_{\Delta t}$ is the shift in time by $\Delta t$ to the left, defined in (5.4), $\nabla^{\tau_{\Delta t} \eta_{N}}$ is the transformed gradient via the ALE mapping $A_{\tau_{\Delta t} \eta_{N}}$, defined in (3.5), and $v_{N}^{*}, \mathbf{u}_{N}, v_{N}$ and $\eta_{N}$ are defined in (5.1).

Now we can use the convergence results from Proposition 5.1, Theorem 5.2, Lemma 5.5 and Corollary 5.1, and arguments analogous to those in [43], to pass to the limit in (6.2) to obtain the following main result:

Theorem 6.1. Let $\rho_{f}, \rho_{K}, \mu_{F}, h, \mu, \lambda>0$. Suppose that the initial data $v_{0} \in L^{2}(\omega), \mathbf{u}_{0} \in L^{2}\left(\Omega_{\eta_{0}}\right)$, and $\eta_{0} \in H_{0}^{2}(\omega)$ are such that $\left(R+\eta_{0}(z, \theta)\right)>0$, $(z, \theta) \in \omega$. Furthermore, let $P_{\text {in }}, P_{\text {out }} \in L_{\text {loc }}^{2}(0, \infty)$.

Then there exist a $T>0$ and a weak solution $(\mathbf{u}, \eta)$ of Problem 3.1 on $(0, T)$ in the sense of Definition 3.1, that satisfy the following energy estimate:

$$
E(t)+\int_{0}^{t} D(\tau) d \tau \leq E_{0}+C\left(\left\|P_{\text {in }}\right\|_{L^{2}(0, t)}^{2}+\left\|P_{\text {out }}\right\|_{L^{2}(0, t)}^{2}\right), \quad t \in[0, T],
$$

where $C$ depends only on the coefficients in the problem, $E_{0}$ is the kinetic energy of initial data, and $E(t)$ and $D(t)$ are given by

$$
\begin{aligned}
& E(t)=\frac{\rho_{f}}{2}\|\mathbf{u}\|_{L^{2}\left(\Omega_{\eta}(t)\right)}^{2}+\frac{\rho_{K} h}{2}\left\|\partial_{t} \eta\right\|_{L^{2}(\omega)}^{2}+\frac{h}{4}\|\eta\|_{\gamma}^{2}+\frac{h^{3}}{48}\|\eta\|_{\sigma}^{2}, \\
& D(t)=\mu_{F}\|\mathbf{D}(\mathbf{u})\|_{\left.L^{2}\left(\Omega_{\eta}(t)\right)\right)}^{2},
\end{aligned}
$$

where

$$
\|f\|_{\gamma}:=\int_{\omega} \mathcal{A} \gamma(f): \gamma(f) R, \quad\|f\|_{\sigma}:=\int_{\omega} \mathcal{A} \boldsymbol{\sigma}(f): \boldsymbol{\sigma}(f) R,
$$


are the energy norms defined by the membrane and flexural effects of the linearly elastic Koiter shell, where the elasticity operator $\mathcal{A}$ is defined in (2.2). They are equivalent to the standard $L^{2}(\omega)$ and $H_{0}^{2}(\omega)$ norms, respectively.

Furthermore, one of the following is true: either

(1) $T=\infty$, or

(2) $\lim _{t \rightarrow T} \min _{z \in[0, L]}(R+\eta(z))=0$.

Proof. It only remains to prove the last assertion, which states that our result is either global in time, or, in case the walls of the cylinder touch each other, our existence result holds until the time of touching. However, the proof of this argument follows the same reasoning as the proof of the Main Theorem in [43], and the proof of the main result in [9], p. 397-398. We avoid repeating those arguments here, and refer the reader to references $[43,9]$.

\section{Conclusions}

We proved the existence of a weak solution to a 3D FSI problem between an incompressible, viscous Newtonian fluid, and a linearly elastic cylindrical Koiter shell, allowing non-radially symmetric shell displacements. The coupled FSI problem is defined on a cylindrical domain, and is driven by the inlet and outlet dynamic pressure data. The main novelties are the following: the existence proof is constructive, it relies on a splitting algorithm used in the design of a computational scheme applied so far to $2 \mathrm{D}$ radiallysymmetric problems [7, 43], and the proof in this manuscript is designed for a full 3D problem, without requiring radial symmetry of the structure displacement. New ideas had to be developed to extend the result from the $2 \mathrm{D}$ radially-symmetric case, to the full $3 \mathrm{D}$ problem. They include:

- A different ALE mapping, and a different discretization of the corresponding Jacobian had to be used to obtain a discretized problem which satisfies the geometric conservation law property, and a discrete energy estimate that mimics the energy estimate of the continuous FSI problem. This part was crucial for the existence proof.

- The fluid-structure interface $\Gamma$ associated with the weak solution of the $3 \mathrm{D}$ problem is not Lipschitz, which gives rise to the various difficulties related to the handling of the function spaces and making sense of the trace of the fluid velocity on $\Gamma$. 
- Different Sobolev embedding theorems had to be used than those used in the $2 \mathrm{D}$ case, which implies different compactness arguments to show strong convergence of approximate sequences.

- In contrast with the $2 \mathrm{D}$ case where we were able to prove the existence of a weak solution by considering a FSI problem in which the structure had the lowest possible regularity, presented by the linear wave equation, in this manuscript we had to include both the membrane and flexural effects in the structure model (i.e., we had to include the 4-th order spatial derivatives and consider the full Koiter shell model) to obtain the existence of a weak solution of the coupled FSI problem. The full Koiter shell model provided enough regularity to make sense of the trace of the fluid velocity on $\Gamma$, and to obtain a weak solution in which the fluid-structure interface is at least Hölder continuous.

The results presented in this manuscript show that the numerical scheme based on the operator splitting approach constructed in this paper, converges to a weak solution of the coupled FSI problem.

While the reference configuration for the fluid domain considered in this manuscript is still a uniform cylinder of radius $R$ and length $L$, we believe that the generalization to having an arbitrary, non-radially symmetric cylinder as a reference configuration (pre-stressed or not) does not present significant mathematical differences with respect to the problem presented in this manuscript. The Koiter shell model in that case, however, is significantly more complicated (see, e.g., [48]), and the calculations in the proof more involving. However, to study the influence of the non-radially symmetric geometry of a native stenosed artery on the behavior of cardiovascular devices such as stents, would require a numerical implementation of the full, non-radially symmetric model, in which the reference configuration is not radially symmetric, but rather it models the geometry of a given stenosed artery.

\section{Acknowledgements}

The authors would like to thank the referee for his/her very insightful comments, which have improved the quality of the manuscript. Further thanks are extended to Martina Bukač, Roland Glowinski, Annalisa Quaini, and Josip Tambača, for inspiring conversations and discussions which influenced, in part, this work. Special thanks are also extended to the National Science Foundation for partial funding of this research via the following grants: NIGMS DMS-1263572, DMS-1318763, DMS-1311709, DMS-1262385, and DMS-1109189. 


\section{References}

[1] R. A. Adams. Sobolev spaces. Academic Press [A subsidiary of Harcourt Brace Jovanovich, Publishers], New York-London, 1975. Pure and Applied Mathematics, Vol. 65. MR0450957

[2] V. Barbu, Z. Grujić, I. Lasiecka, and A. Tuffaha. Existence of the energy-level weak solutions for a nonlinear fluid-structure interaction model. In Fluids and waves, volume 440 of Contemp. Math., pages 55-82. Amer. Math. Soc., Providence, RI, 2007. MR2359449

[3] V. Barbu, Z. Grujić, I. Lasiecka, and A. Tuffaha. Smoothness of weak solutions to a nonlinear fluid-structure interaction model. Indiana Univ. Math. J., 57(3):1173-1207, 2008. MR2429089

[4] H. Beirão da Veiga. On the existence of strong solutions to a coupled fluid-structure evolution problem. J. Math. Fluid Mech., 6(1):21-52, 2004. MR2027753

[5] M. Boulakia. Existence of weak solutions for the motion of an elastic structure in an incompressible viscous fluid. C. R. Math. Acad. Sci. Paris, 336(12):985-990, 2003. MR1993967

[6] M. Bukač, P. Zunino, and I. Yotov. Explicit partitioning strategies for interaction of the fluid with a multilayered poroelastic structure: An operator-splitting approach. Submitted, 2013.

[7] M. Bukac, S. Canic, R. Glowinski, J. Tambaca, and A. Quaini. Fluidstructure interaction in blood flow capturing non-zero longitudinal structure displacement. Journal of Computational Physics, 235(0):515541, 2013. MR3017610

[8] P. Causin, J. F. Gerbeau, and F. Nobile. Added-mass effect in the design of partitioned algorithms for fluid-structure problems. Comput. Methods Appl. Mech. Engrg., 194(42-44):4506-4527, 2005. MR2157973

[9] A. Chambolle, B. Desjardins, M. J. Esteban, and C. Grandmont. Existence of weak solutions for the unsteady interaction of a viscous fluid with an elastic plate. J. Math. Fluid Mech., 7(3):368-404, 2005. MR2166981

[10] C. H. A. Cheng, D. Coutand, and S. Shkoller. Navier-Stokes equations interacting with a nonlinear elastic biofluid shell. SIAM J. Math. Anal., 39(3):742-800 (electronic), 2007. MR2349865 
[11] C. H. A. Cheng and S. Shkoller. The interaction of the 3D NavierStokes equations with a moving nonlinear Koiter elastic shell. SIAM J. Math. Anal., 42(3):1094-1155, 2010. MR2644917

[12] C. H. Ciarlet and D. Coutand. An existence theorem for nonlinearly elastic "flexural" shells. J. Elasticity, 50(3):261-277, 1998. MR1651342

[13] C. R. Ciarlet and A. Roquefort. Justification of a two-dimensional shell model of koiter type. C.R. Acad. Sci. Paris, Ser I Math. 331(5):411416, 2000. MR1784924

[14] P. G. Ciarlet. A two-dimensional nonlinear shell model of koiter type. C.R. Acad. Sci. Paris. Ser I Math., 331:405-410, 2000. MR1784923

[15] C. Conca, F. Murat, and O. Pironneau. The Stokes and Navier-Stokes equations with boundary conditions involving the pressure. Japan. J. Math. (N.S.), 20(2):279-318, 1994. MR1308419

[16] C. Conca, J. San Martín H., and M. Tucsnak. Motion of a rigid body in a viscous fluid. C. R. Acad. Sci. Paris Sér. I Math., 328(6):473-478, 1999. MR1680008

[17] D. Coutand and S. Shkoller. Motion of an elastic solid inside an incompressible viscous fluid. Arch. Ration. Mech. Anal., 176(1):25-102, 2005. MR2185858

[18] D. Coutand and S. Shkoller. The interaction between quasilinear elastodynamics and the Navier-Stokes equations. Arch. Ration. Mech. Anal., 179(3):303-352, 2006. MR2208319

[19] P. Cumsille and T. Takahashi. Wellposedness for the system modelling the motion of a rigid body of arbitrary form in an incompressible viscous fluid. Czechoslovak Math. J., 58(133)(4):961-992, 2008. MR2471160

[20] B. Desjardins and M. J. Esteban. Existence of weak solutions for the motion of rigid bodies in a viscous fluid. Arch. Ration. Mech. Anal., 146(1):59-71, 1999. MR1682663

[21] B. Desjardins, M. J. Esteban, C. Grandmont, and P. Le Tallec. Weak solutions for a fluid-elastic structure interaction model. Rev. Mat. Complut., 14(2):523-538, 2001. MR1871311

[22] J. Donea. Arbitrary lagrangian-eulerian finite element methods, in: Computational methods for transient analysis. North-Holland, Amsterdam, 1983. 
[23] Q. Du, M. D. Gunzburger, L. S. Hou, and J. Lee. Analysis of a linear fluid-structure interaction problem. Discrete Contin. Dyn. Syst., 9(3):633-650, 2003. MR1974530

[24] C. Farhat, P. Geuzaine, and C. Grandmont. The discrete geometric conservation law and the nonlinear stability of ale schemes for the solution of flow problems on moving grids. Journal of Computational Physics, 174(2):669-694, 2001. MR1868101

[25] E. Feireisl. On the motion of rigid bodies in a viscous compressible fluid. Arch. Ration. Mech. Anal., 167(4):281-308, 2003. MR1981859

[26] G. P. Galdi. Mathematical problems in classical and non-Newtonian fluid mechanics. In Hemodynamical flows, volume 37 of Oberwolfach Semin., pages 121-273. Birkhäuser, Basel, 2008. MR2410706

[27] R. Glowinski. Finite element methods for incompressible viscous flow, in: P. G. Ciarlet, J.-L. Lions (Eds), Handbook of numerical analysis, volume 9. North-Holland, Amsterdam, 2003. MR2009826

[28] C. Grandmont. Existence of weak solutions for the unsteady interaction of a viscous fluid with an elastic plate. SIAM J. Math. Anal., 40(2):716737, 2008. MR2438783

[29] G. Guidoboni, M. Guidorzi, and M. Padula. Continuous dependence on initial data in fluid-structure motions. J. Math. Fluid Mech., 14(1):132, 2012. MR2891187

[30] G. Guidoboni, R. Glowinski, N. Cavallini, and S. Canic. Stable looselycoupled-type algorithm for fluid-structure interaction in blood flow. $J$. Comput. Phys., 228(18):6916-6937, 2009. MR2567876

[31] A. Hundertmark-Zaušková, M. Lukáčová-Medvidová, and G. Rusnáková. Fluid-structure interaction for shear-dependent non-Newtonian fluids. In Topics in mathematical modeling and analysis, volume 7 of Jindr̆ich Nečas Cent. Math. Model. Lect. Notes, pages 109-158. Matfyzpress, Prague, 2012. MR2962823

[32] W. T. Koiter. On the foundations of the linear theory of thin elastic shells. i, ii. Nederl. Akad. Wetensch. Proc., Ser. B 73. MR0280050

[33] I. Kukavica and A. Tuffaha. Solutions to a fluid-structure interaction free boundary problem. DCDS-A, 32(4):1355-1389, 2012. MR2851902

[34] I. Kukavica and A. Tuffaha. Well-posedness for the compressible NavierStokes-Lamé system with a free interface. Nonlinearity, 25(11):31113137, 2012. MR2991431 
[35] I. Kukavica, A. Tuffaha, and M. Ziane. Strong solutions for a fluid structure interaction system. Adv. Differential Equations, 15(3-4):231254, 2010. MR2588449

[36] D. Lengeler. Global weak solutions for an incompressible, generalized newtonian fluid interacting with a linearly elastic koiter shell. arXiv:1212.3435, 2012.

[37] D. Lengeler and M. Ružička. Global weak solutions for an incompressible newtonian fluid interacting with a linearly elastic koiter shell. arXiv:1207.3696v1, 2012. MR3147436

[38] J. Lequeurre. Existence of strong solutions to a fluid-structure system. SIAM J. Math. Anal., 43(1):389-410, 2011. MR2765696

[39] J. Lequeurre. Existence of Strong Solutions for a System Coupling the Navier-Stokes Equations and a Damped Wave Equation. J. Math. Fluid Mech., 15(2):249-271, 2013. MR3061763

[40] A. Hundertmark-Zaušková, M. Lukáčová-Medvid'ová, G. Rusnáková. Kinematic splitting algorithm for fluid-structure interaction in hemodynamics. Computer Methods in Appl. Mech. Engi., To appear., 2013. MR3093603

[41] B. Muha. A note on the trace theorem for domains which are locally subgraph of Hölder continuous function. Netw. Heterog. Media, 9(1):191-196, 2014. MR3195351

[42] B. Muha and S. Čanić. Existence of a solution to a fluid-multi-layeredstructure interaction problem. J. Differential Equations, 256(2):658706, 2014. MR3121710

[43] B. Muha and S. Canić. Existence of a Weak Solution to a Nonlinear Fluid-Structure Interaction Problem Modeling the Flow of an Incompressible, Viscous Fluid in a Cylinder with Deformable Walls. Arch. Ration. Mech. Anal., 207(3):919-968, 2013. MR3017292

[44] A. Quarteroni, M. Tuveri, and A. Veneziani. Computational vascular fluid dynamics: problems, models and methods. Comput. Vis. Sci., 2(4):163-197, 2000.

[45] A. Quarteroni, M. Tuveri, and A. Veneziani. Computational vascular fluid dynamics: problems, models and methods. survey article. Comput. Visual. Sci., 2:163-197, 2000.

[46] J. A. San Martín, V. Starovoitov, and M. Tucsnak. Global weak solutions for the two-dimensional motion of several rigid bodies in an in- 
compressible viscous fluid. Arch. Ration. Mech. Anal., 161(2):113-147, 2002. MR1870954

[47] J. Simon. Compact sets in the space $L^{p}(0, T ; B)$. Ann. Mat. Pura Appl. (4), 146:65-96, 1987. MR0916688

[48] J. Tambača, S. Čanić, and A. Mikelić. Effective model of the fluid flow through elastic tube with variable radius. Grazer Mathematische Berichte, 348, 2005. MR2228391

[49] R. Temam. Sur la résolution exacte et approchée d'un problème hyperbolique non linéaire de T. Carleman. Arch. Rational Mech. Anal., 35:351-362, 1969. MR0251376

[50] S. Čanić, B. Muha, and M. Bukač. Stability of the kinematically coupled $\beta$-scheme for fluid-structure interaction problems in hemodynamics. Accepted, arXiv:1205.6887, 2012.

[51] I. Velčić. Nonlinear weakly curved rod by $\Gamma$-convergence. J. Elasticity, 108(2):125-150, 2012. MR2948053

Boris MuHA

Department of Mathematics

UNIVERSITY OF ZAGREB

Croatia

E-mail address: borism@math.hr

SUNČICA ČAnIĆ

Department of Mathematics

UNIVERSITY OF HOUSTON

Houston, TX 77204

USA

E-mail address: canic@math.uh.edu

Received October 2, 2013 